\title{
High-order Discontinuous Galerkin methods for the elastodynamics equation on polygonal and polyhedral meshes ${ }^{\text {th }}$
}

\author{
P.F. Antonietti ${ }^{\mathrm{a}}$, I. Mazzieri ${ }^{\mathrm{a}, *}$ \\ ${ }^{a}$ MOX-Laboratory for Modeling and Scientific Computing, Dipartimento di Matematica, Politecnico di Milano, \\ Piazza Leonardo da Vinci 32, 20133 Milano, Italy
}

\begin{abstract}
We propose and analyze a high-order Discontinuous Galerkin Finite Element Method for the approximate solution of wave propagation problems modeled by the elastodynamics equations on computational meshes made by polygonal and polyhedral elements. We analyze the well posedness of the resulting formulation, prove $h p$-version error a-priori estimates, and present a dispersion analysis, showing that polygonal meshes behave as classical simplicial/quadrilateral grids in terms of dispersion properties. The theoretical estimates are confirmed through various two-dimensional numerical verifications.
\end{abstract}

Keywords: Discontinuous Galerkin methods, elastodynamics, wave propagation, polygonal and polyhedral grids

2010 MSC: 65M12, 65M60

\section{Introduction}

The study of direct and inverse wave propagation phenomena is an intensive research area, stimulated by geophysical, aeroacoustic, acoustics, and electromagnetics applications. One important field of application includes large-scale seismological problems and ground-motion induced by seismic events. Seismic waves are elastic waves propagating within the earth and along its surface as a result of an earthquake or an explosion, and induce a vibratory ground-motion in the area surrounding the seismic source. From the mathematical perspective, the propagation of seismic waves in an elastic material can be modeled by means of the elastodynamics equation. From the numerical viewpoint, a number of distinguishing challenges arise when tackling such kind of problems, and reflect onto the following features required to the numerical schemes: accuracy, geometric flexibility and scalability. High-order accuracy is mandatory in order to keep numerical dissipation and dispersion low. Geometric flexibility is required since the computational domain

\footnotetext{
is The authors have been partially supported by SIR Starting grant n. RBSI14VT0S funded by MIUR - Italian Ministry of Education, Universities and Research. This work was partially supported by "National Group of Computing Science" (GNCS-INdAM).

* Corresponding author

Email addresses: paola.antonietti@polimi.it (P.F. Antonietti), ilario.mazzieri@polimi.it (I. Mazzieri)
} 
usually features complicated geometric details as well as sharp contrasts in the media. Additionally, for real earthquake models the size of the excited body is very large compared to the wave lengths of interest. This typically leads to a discrete (linear) system of equations with several millions of unknowns, and therefore massively parallel scalable algorithms are needed.

Spectral element methods are one of the most successful tool in computational seismology, in particular for large scale applications, see for example [1, 2]. Another numerical method that in recent years has been extensively used for elastic wave propagation is the Discontinuous Galerkin (DG) method, see e.g. [3, 4, 5, 6, 7, 8, 9, 10, 11, 12, 13, . We refer to 14, 15, 16, for a general overview on DG methods. Thanks to their local nature, DG methods are particularly apt to treat highly heterogeneous and soil-structure interaction problems, where local refinements are needed to resolve the different spatial scales. Recently, DG methods have been shown to be naturally well suited to handle meshes composed by arbitrarily-shaped polygonal/polyhedral (polytopic, for short) elements, see e.g. [17, 18, 19, 20, 21, 22, 23, 24, 25, 26, 27, 28]. The flexibility in the process of mesh design offered by polytopic elements is a great advantage whenever the differential problem at hand is posed on complicated domains featuring internal layers, microstructures, fractures or heterogeneities, as for example in geophysical applications, fluid-structure interaction or crack propagation problems. The versatility offered by polygonal/polyhedral meshes has boosted in recent years an intensive development of numerical methods for partial differential equations that can allow for such kind of decompositions. Beside DG methods on polytopic meshes, in the conforming setting we also mention, for example, the Virtual Element Method ([29, 30, 31, 32, 33, 34]), the Mimetic Finite Difference Method ([35, 36, 37, 38, 39, 40]), the Composite Finite Element Method (41, 42, 18]), the Extended Finite Element Method (43, 44]), the Polygonal Finite Element 35 Method (45]), and the Hybrid High-Order method (46, 47, 48, 49]). The aim of this paper is to approximate the elastodynamics equations with a the high-order Discontinuous Galerkin method on polygonal/polyhedral decompositions, providing both a rigorous stability and error estimates, a dispersion analysis as well as numerical simulations. We show that the resulting formulation satisfies $h p$-version error estimates in a suitable mesh-dependent energy norm, and show that the present formulation guarantees lower dispersion errors compared to classical DG schemes on simplicial/quadrilateral grids of comparable granularity. The theoretical results are supported by numerical experiments on both benchmark and real test cases.

To the best of our knowledge, even if some techniques used to prove our main theorems can be found in previous works, as for instance [10, 13, the results shown in this paper have not appeared elsewhere in its present form. Moreover it is the first time that such a discretization is applied in the framework of elastic wave propagation problems.

The remaining part of the paper is organized as follows. In Section 2 we introduce the equilibrium equations for an elastic medium and its high-order DG approximation on polygo- 
nal/polyhedral grids. Section 3 is devoted to the proof of the stability bounds as well as of $h p$-version error estimates for the semidiscrete scheme. The algebraic formulation and time integration is discussed in Section 4 whereas the dispersion analysis in presented in Section 5 . Finally, some numerical results are shown in Section [6. In Section 7 we draw some conclusions.

\section{Model problem and its discretization}

We use standard notation for the Sobolev spaces $H^{m}(\Omega), m \geq 0$, endowed with the usual norm $\|\cdot\|_{H^{m}(\Omega)}$ and seminorm $|\cdot|_{H^{m}(\Omega)}$, cf. [50]. For $m=0$ we denote the corresponding space by $L^{2}(\Omega)$ and the associated norm by $\|\cdot\|_{0, \Omega}$. We denote the corresponding Sobolev spaces of vectorvalued functions and symmetric tensors by $\boldsymbol{H}^{m}(\Omega)=\left[H^{m}(\Omega)\right]^{d}, \mathcal{H}^{m}(\Omega)=\left[H^{m}(\Omega)\right]_{\text {sym }}^{d \times d}, d=2,3$, respectively. We consider an elastic body occupying an open, bounded convex polyhedral domain $\Omega \subset \mathbb{R}^{d}, d=2,3$, and denote by $\Gamma=\partial \Omega$ its boundary with outward normal unit vector $\mathbf{n}$. The boundary is assumed to be composed of two disjoint portions $\Gamma_{D} \neq \emptyset$ and $\Gamma_{N}$, i.e., $\Gamma_{D} \cap \Gamma_{N}=\emptyset$. Given a suitable external load $\mathbf{f} \in L^{2}\left((0, T] ; \mathbf{L}^{2}(\Omega)\right)$, and suitable initial/boundary data $\mathbf{g} \in$ $C^{1}\left((0, T] ; \mathbf{H}^{\frac{1}{2}}\left(\Gamma_{N}\right)\right), \mathbf{u}_{0} \in \mathbf{H}_{0, \Gamma_{D}}^{1}(\Omega)$ and $\mathbf{u}_{1} \in \mathbf{L}^{2}(\Omega)$, the equations of (linear) elastodynamics are given by

$$
\left\{\begin{array}{rlrl}
\rho \ddot{\mathbf{u}}-\nabla \cdot \boldsymbol{\sigma}=\mathbf{f}, & & \text { in } \Omega \times(0, T], \\
\mathbf{u} & =\mathbf{0}, & & \text { on } \Gamma_{D} \times(0, T], \\
\boldsymbol{\sigma} \mathbf{n} & =\mathbf{g}, & & \text { on } \Gamma_{N} \times(0, T], \\
\mathbf{u} & =\mathbf{u}_{0}, & & \text { in } \Omega \times\{0\}, \\
\dot{\mathbf{u}} & =\mathbf{u}_{1}, & & \text { in } \Omega \times\{0\} .
\end{array}\right.
$$

where the displacement vector is denoted by $\mathbf{u}: \Omega \times[0, T] \rightarrow \mathbb{R}^{d}$, being $[0, T]$ the time interval with $T>0$. Denoting by $\mathbb{S}$ the space of symmetric, $d \times d$, real-valued tensorial functions, $d=2,3$, we assume the generalized Hooke's law for the stress tensor $\boldsymbol{\sigma}: \Omega \times[0, T] \rightarrow \mathbb{S}$, i.e., $\boldsymbol{\sigma}(\mathbf{u})=\mathcal{D} \varepsilon(\mathbf{u})$, where the fourth order stiffness tensor $\mathcal{D}: \mathbb{S} \rightarrow \mathbb{S}$ is defined as

$$
\mathcal{D} \boldsymbol{\tau}=2 \mu \boldsymbol{\tau}+\lambda \operatorname{tr}(\boldsymbol{\tau}) \mathbf{I} \quad \forall \boldsymbol{\tau} \in \mathbb{S},
$$

and $\varepsilon(\mathbf{u})$ is the symmetric gradient of $\mathbf{u}$, i.e., $\varepsilon(\mathbf{u})=\frac{1}{2}\left(\nabla \mathbf{u}+\nabla \mathbf{u}^{T}\right)$. In 22, I is the identity tensor, $\operatorname{tr}(\cdot)$ represents the trace operator, while $\lambda, \mu \in L^{\infty}(\Omega)$ are the first and the second Lamé parameters, respectively. We assume that $\mathcal{D}$ is symmetric, positive definite and uniformly bounded over $\Omega$. The compressional $(\mathrm{P})$ and shear $(\mathrm{S})$ wave velocities of the medium are obtained through the relations $c_{P}=\sqrt{(\lambda+2 \mu) / \rho}$ and $c_{S}=\sqrt{\mu / \rho}$, respectively.

The weak formulation of problem (1) reads as follows: for all $t \in(0, T]$ find $\mathbf{u}=\mathbf{u}(t) \in \mathbf{H}_{0, \Gamma_{D}}^{1}(\Omega)$ 
such that:

$$
\left\{\begin{array}{l}
\int_{\Omega} \rho \ddot{\mathbf{u}} \cdot \mathbf{v} d \mathbf{x}+\int_{\Omega} \mathcal{D} \varepsilon(\mathbf{u}): \varepsilon(\mathbf{v}) d \mathbf{x}=\int_{\Omega} \mathbf{f} \cdot \mathbf{v} d \mathbf{x}+\int_{\Gamma_{N}} \mathbf{g} \cdot \mathbf{v} d \mathbf{s} \quad \forall \mathbf{v} \in \mathbf{H}_{0, \Gamma_{D}}^{1}(\Omega), \\
\mathbf{u}(0)=\mathbf{u}_{0} \quad \dot{\mathbf{u}}(0)=\mathbf{u}_{1} .
\end{array}\right.
$$

60 Problem (3) is well posed and its unique solution $\mathbf{u} \in C\left((0, T] ; \mathbf{H}_{0, \Gamma_{D}}^{1}(\Omega)\right) \cap C^{1}\left((0, T] ; \mathbf{L}^{2}(\Omega)\right)$, see [51, Theorem 8-3.1].

\subsection{DG formulation}

In the following we introduce the high-order DG approximation of problem (3).

Polygonal and polyhedral meshes. Let $\mathcal{T}$ be a non-overlapping partition of the domain $\Omega$ made by open disjoint polygonal/polyhedral elements $\kappa$ of diameter $h_{\kappa}$ such that $\bar{\Omega}=\bigcup_{\kappa \in \mathcal{T}} \bar{\kappa}$. We define the faces of the mesh $\mathcal{T}$ as the planar/straight intersection of the $(d-1)$-dimensional facets of neighboring elements. This implies that, for $d=2$, a face always consists of a line segment, while for $d=3$, the faces of $\mathcal{T}$ are general shaped polygons, which we assume can be further subdivided into a set of co-planar triangles. In three-dimension, with a slightly abuse of notation, we then use the terminology face to refer to a $(d-1)$-dimensional simplex which forms part of the interface of an element. Let $\mathcal{F}=\mathcal{F}_{I} \cup \mathcal{F}_{B}$ be the union of all interior and boundary faces. Moreover, we set $\mathcal{F}_{B}=\mathcal{F}_{D} \cup \mathcal{F}_{N}$ where $\mathcal{F}_{D}=\left\{F \in \mathcal{F}_{B}: F \subseteq \Gamma_{D}\right\}$ and $\mathcal{F}_{N}=\left\{F \in \mathcal{F}_{B}: F \subseteq \Gamma_{N}\right\}$. Implicit in this definition is the assumption that $\mathcal{T}$ respects the decomposition of $\partial \Omega$, that is, any $\gamma \in \mathcal{F}_{B}$ belongs to the interior of exactly one of $\mathcal{F}_{D}$ or $\mathcal{F}_{N}$.

Finite element spaces. Denoting by $\mathcal{P}_{p_{\kappa}}(\kappa)$ the space of polynomials of total degree at most $p_{\kappa} \geq 1$ on $\kappa \in \mathcal{T}$, we define the finite element spaces $\mathrm{V}_{h p}, \mathbf{V}_{h p}$ and $\mathcal{V}_{h p}$ as

$$
\mathrm{V}_{h p}=\left\{v \in L^{2}(\Omega):\left.v\right|_{\kappa} \in \mathcal{P}_{p_{\kappa}}(\kappa) \quad \forall \kappa \in \mathcal{T}\right\}, \quad \mathbf{V}_{h p}=\left[\mathrm{V}_{h p}\right]^{d}, \quad \mathcal{V}_{h p}=\left[\mathrm{V}_{h p}\right]_{\mathrm{sym}}^{d \times d}, \quad d=2,3,
$$

respectively.

Trace operators. For (regular enough) vector-valued and tensor-valued functions $\mathbf{v}$ and $\boldsymbol{\tau}$, respectively, on each interior face $\gamma \in \mathcal{F}_{I}$ shared by two adjacent elements $\kappa^{ \pm} \in \mathcal{T}$ with outward 
unit normal vectors $\mathbf{n}_{\kappa}^{ \pm}$, we define the average and jump operators

$$
\begin{array}{rlrl}
\{\mathbf{v}\} & =\frac{1}{2}\left(\mathbf{v}^{+}+\mathbf{v}^{-}\right), & & \llbracket \mathbf{v} \rrbracket=\mathbf{v}^{+} \odot \mathbf{n}_{\kappa}^{+}+\mathbf{v}^{-} \odot \mathbf{n}_{\kappa}^{-}, \\
\{\boldsymbol{\tau}\}=\frac{1}{2}\left(\boldsymbol{\tau}^{+}+\boldsymbol{\tau}^{-}\right), & \llbracket \boldsymbol{\tau} \rrbracket=\boldsymbol{\tau}^{+} \mathbf{n}_{\kappa}^{+}+\boldsymbol{\tau}^{-} \mathbf{n}_{\kappa}^{-},
\end{array}
$$

80

where $\mathbf{v} \odot \mathbf{n}_{\kappa}=\left(\mathbf{v n}_{\kappa}^{T}+\mathbf{n}_{\kappa} \mathbf{v}^{T}\right) / 2$. On a boundary face $\gamma \in \mathcal{F}_{B}$ we set analogously $\{\mathbf{v}\}=\mathbf{v}^{+}$, $\llbracket \mathbf{v} \rrbracket=\mathbf{v}^{+} \odot \mathbf{n},\{\boldsymbol{\tau}\}=\boldsymbol{\tau}^{+}$, and $\llbracket \boldsymbol{\tau} \rrbracket=\boldsymbol{\tau}^{+} \mathbf{n}$, cf. [52, 53. Notice that with the above definitions $\llbracket \mathbf{v} \rrbracket$ is a $d \times d$ symmetric tensor.

Semidiscrete DG formulation. The semidiscrete DG formulation reads as: $\forall t \in(0, T]$ find $\mathbf{u}_{h}=\mathbf{u}_{h}(t) \in \mathbf{V}_{h p}$ such that

$$
\int_{\Omega} \rho \ddot{\mathbf{u}}_{h} \cdot \mathbf{v} d \mathbf{x}+\mathcal{B}\left(\mathbf{u}_{h}, \mathbf{v}\right)=\int_{\Omega} \mathbf{f} \cdot \mathbf{v} d \mathbf{x}+\int_{\mathcal{F}_{N}} \mathbf{g} \cdot \mathbf{v} d \mathbf{s} \quad \forall \mathbf{v} \in \mathbf{V}_{h p}
$$

supplemented with the initial conditions $\mathbf{u}_{h}(0)=\mathbf{u}_{h}^{0}$ and $\dot{\mathbf{u}}_{h}(0)=\mathbf{u}_{h}^{1}, \mathbf{u}_{h}^{0}, \mathbf{u}_{h}^{1} \in \mathbf{V}_{h p}$ being suitable approximations of $\mathbf{u}_{0}$ and $\mathbf{u}_{1}$, respectively. The bilinear form $\mathcal{B}(\cdot, \cdot): \mathbf{V}_{h p} \times \mathbf{V}_{h p} \rightarrow \mathbb{R}$ is defined as

$$
\begin{aligned}
\mathcal{B}(\mathbf{u}, \mathbf{v})=\sum_{\kappa \in \mathcal{T}} \int_{\kappa} \boldsymbol{\sigma}(\mathbf{u}): \boldsymbol{\varepsilon}(\mathbf{v}) d \mathbf{x}-\int_{\mathcal{F}_{I} \cup \mathcal{F}_{D}}\{\boldsymbol{\sigma}(\mathbf{u})\}: \llbracket \mathbf{v} \rrbracket d \mathbf{s} \\
-\int_{\mathcal{F}_{I} \cup \mathcal{F}_{D}} \llbracket \mathbf{u} \rrbracket:\{\boldsymbol{\sigma}(\mathbf{v})\} d \mathbf{s}+\int_{\mathcal{F}_{I} \cup \mathcal{F}_{D}} \eta \llbracket \mathbf{u} \rrbracket: \llbracket \mathbf{v} \rrbracket d \mathbf{s} \quad \forall \mathbf{u}, \mathbf{v} \in \mathbf{V}_{h p},
\end{aligned}
$$

with the short-hand notation $\int_{\mathcal{F}_{I} \cup \mathcal{F}_{D}} \psi d \mathbf{s}=\sum_{\gamma \in \mathcal{F}_{I} \cup \mathcal{F}_{D}} \int_{\gamma} \psi d \mathbf{s}$. For the sake of the analysis, we write problem (4) in the following equivalent form: given $\mathbf{u}_{h}^{0}, \mathbf{u}_{h}^{1} \in \mathbf{V}_{h p}, \forall t \in(0, T]$ find $\mathbf{u}_{h}=\mathbf{u}_{h}(t) \in \mathbf{V}_{h p}$ such that

$$
\int_{\Omega} \rho \ddot{\mathbf{u}}_{h} \cdot \mathbf{v} d \mathbf{x}+\widetilde{\mathcal{B}}\left(\mathbf{u}_{h}, \mathbf{v}\right)=\int_{\Omega} \mathbf{f} \cdot \mathbf{v} d \mathbf{x}+\int_{\mathcal{F}_{N}} \mathbf{g} \cdot \mathbf{v} d \mathbf{s}
$$

for all $\mathbf{v} \in \mathbf{V}_{h p}$, where

$$
\begin{aligned}
\widetilde{\mathcal{B}}(\mathbf{u}, \mathbf{v})=\sum_{\kappa \in \mathcal{T}} \int_{\kappa} \boldsymbol{\sigma}(\mathbf{u}): \varepsilon(\mathbf{v}) d \mathbf{x}+\int_{\Omega} \boldsymbol{\sigma}(\mathbf{u}): & \mathcal{R}(\llbracket \mathbf{v} \rrbracket) d \mathbf{x} \\
& +\int_{\Omega} \mathcal{R}(\llbracket \mathbf{u} \rrbracket): \boldsymbol{\sigma}(\mathbf{v}) d \mathbf{x}+\int_{\mathcal{F}_{I} \cup \mathcal{F}_{D}} \eta \llbracket \mathbf{u} \rrbracket: \llbracket \mathbf{v} \rrbracket d \mathbf{s},
\end{aligned}
$$

for all $\mathbf{u}, \mathbf{v} \in \mathbf{V}_{h p}$. Here, $\mathcal{R}(\cdot): \mathcal{L}^{2}\left(\mathcal{F}_{I} \cup \mathcal{F}_{D}\right) \rightarrow \mathcal{V}_{h p}$ is the lifting operator of the traces of $d \times d$ 
symmetric tensors defined as

$$
\int_{\Omega} \mathcal{R}(\llbracket \mathbf{w} \rrbracket): \boldsymbol{\sigma}(\mathbf{v}) d \mathbf{x}=-\int_{\mathcal{F}_{I} \cup \mathcal{F}_{D}} \llbracket \mathbf{w} \rrbracket:\{\boldsymbol{\sigma}(\mathbf{v})\} d \mathbf{s} \quad \forall \mathbf{v} \in \mathcal{V}_{h p}
$$

We note that, despite formulations (4) and (6) are equivalent at the discrete level, formulation (6) ${ }_{85}$ is not strongly consistent with the continuous problem due to the discrete nature of the lifting operator (8).

Supposing that $\mathcal{D}$ is piecewise constant over mesh-elements, in (5) and (7), the penalization parameter $\eta: \mathcal{F} \rightarrow \mathbb{R}_{+}$is defined as

$$
\eta(\mathbf{x})= \begin{cases}C_{\eta} \max _{\kappa \in\left\{\kappa^{+}, \kappa^{-}\right\}}\left(\overline{\mathcal{D}}_{\kappa} \frac{p_{\kappa}^{2}}{h_{\kappa}}\right), & \mathbf{x} \in \gamma, \gamma \in \mathcal{F}_{I}, \gamma \subset \partial \kappa^{+} \cap \partial \kappa^{-}, \\ C_{\eta} \overline{\mathcal{D}}_{\kappa} \frac{p_{\kappa}^{2}}{h_{\kappa}}, & \mathbf{x} \in \gamma, \gamma \in \mathcal{F}_{D}, \gamma \subset \partial \kappa^{+} \cap \Gamma_{D},\end{cases}
$$

where $\overline{\mathcal{D}}_{\kappa}=\left(\left|\mathcal{D}^{\frac{1}{2}}\right|_{2}^{2}\right)_{\left.\right|_{\kappa}}^{\frac{1}{2}}$ and $|\cdot|_{2}$ is the operator norm induced by the $\ell^{2}$-norm on $\mathbb{R}^{d}, d=2,3$. Here $C_{\eta}$ is a positive constant at our disposal.

\section{Stability and error analysis}

In this section we prove stability estimates with respect to a suitable norm induced by the DG method. For the sake of simplicity, we consider $\mathbf{g}=\mathbf{0}$ on $\Gamma_{N}$. Throughout the analysis, the inequality $a \lesssim b$ means that $a \leq C b$ for a multiplicative positive constant $C$ that might depend on the material properties and the shape regularity constant of the covering $\mathcal{T}^{\sharp}$, cf. Assumption

951 below, but is independent of the discretization parameters, as well as the number of faces per mesh element and the relative measure of the face compared to element diameter. We first recall some preliminary results that will be needed in our theoretical analysis.

\subsection{Preliminary results}

We first introduce the following assumptions on the partition $\mathcal{T}$ that will be needed for the following analysis, see [22] and also [26].

Assumption 1. We assume that the partition $\mathcal{T}$ satisfies

11a) For any $\kappa \in \mathcal{T}$, there exists a set of nonoverlapping (not-necessary shape regular) d-dimensional simplices $T_{\ell} \subseteq \kappa, \ell=1,2, \ldots, n_{\kappa}$, such that, for any face $\gamma \subset \partial \kappa, \bar{\gamma}=\partial \bar{\kappa} \cap \partial \bar{T}_{\ell}$, for some $\ell$, it holds

$$
\bigcup_{\ell=1}^{n_{\kappa}} \bar{T}_{\ell} \subseteq \bar{\kappa}
$$


and the diameter $h_{\kappa}$ of $\kappa$ can be bounded by

$$
h_{\kappa} \lesssim \frac{d\left|T_{\ell}\right|}{|\gamma|}, \quad \ell=1,2, \ldots, n_{\kappa} .
$$

1b) Let $\mathcal{T}^{\sharp}=\{\mathcal{K}\}$ denotes a covering of $\Omega$ consisting of shape-regular d-dimensional simplices $\mathcal{K}$. We assume that, for any $\kappa \in \mathcal{T}$, there exists $\mathcal{K} \in \mathcal{T}^{\sharp}$ such that $\kappa \subset \mathcal{K}, \operatorname{diam}(\mathcal{K}) \lesssim h_{\kappa}$ and

$$
\max _{\kappa \in \mathcal{T}} \operatorname{card}\left\{\kappa^{\prime} \in \mathcal{T}: \kappa^{\prime} \cap \mathcal{K} \neq \emptyset, \mathcal{K} \in \mathcal{T}^{\sharp} \quad \text { such that } \kappa \subset \mathcal{K}\right\} \lesssim 1 \text {. }
$$

We remark that Assumption 1 does not put any restriction on either the number of faces that an element possesses, or the measure of a face of an element $\kappa \in \mathcal{T}$, relative to the measure of the element itself, cf. [22]. Assumption 12) will be required to prove the trace-inverse estimates presented in Lemma 3.1] cf. also [26, whereas Assumption 1b) is needed in view of the approximation result that will be presented in the next section, cf. [22].

We next recall the following trace-inverse inequality for polygonal/polyhedral elements, which is the vectorial counterpart of the analogous one shown in [22], cf. also [20, 26].

Lemma 3.1. Assume that $\mathcal{T}$ satisfies Assumption 1 1a), and let $\kappa \in \mathcal{T}$ be a polygonal/polyhedral element. Then, it holds

$$
\|\boldsymbol{v}\|_{L^{2}(\partial \kappa)}^{2} \lesssim \frac{p_{\kappa}^{2}}{h_{\kappa}}\|\boldsymbol{v}\|_{L^{2}(\kappa)}^{2} \quad \forall \boldsymbol{v} \in\left[\mathcal{P}_{p_{\kappa}}(\kappa)\right]^{d}
$$

From the above lemma we immediately have the following

Lemma 3.2. Let $C_{\eta}$ be the constant appearing in the definition of the penalty function, cf. (9). Then, it holds

$$
\left\|\eta^{-1 / 2}\{\boldsymbol{w}\}\right\|_{0, \mathcal{F}_{I} \cup \mathcal{F}_{D}}^{2} \lesssim \frac{1}{C_{\eta}}\|\boldsymbol{w}\|_{0, \Omega} \quad \forall \boldsymbol{w} \in \mathcal{V}_{h p}
$$

Proof. From the definitions of the average operator and of the penalty parameter $\eta$ given in (9), we have

$$
\eta \geq C_{\eta} \mathcal{D}_{*} \frac{p_{\kappa}^{2}}{h_{\kappa}} \quad \forall \mathbf{x} \in \partial \kappa, \kappa \in \mathcal{T} .
$$

where $\mathcal{D}_{*}$ is the lower bound on $\mathcal{D}$, and therefore

$$
\eta^{-1} \leq \frac{1}{\mathcal{D}_{*} C_{\eta}} \frac{h_{\kappa}}{p_{\kappa}^{2}} \quad \forall \mathbf{x} \in \partial \kappa, \kappa \in \mathcal{T}
$$

Then, using Lemma 3.1, we have

$$
\left\|\eta^{-1 / 2}\{\mathbf{w}\}\right\|_{0, \mathcal{F}_{I} \cup \mathcal{F}_{D}}^{2} \lesssim \sum_{\kappa \in \mathcal{T}}\left\|\eta^{-1 / 2} \mathbf{w}\right\|_{0, \partial \kappa}^{2} \lesssim \frac{1}{C_{\eta}} \sum_{\kappa \in \mathcal{T}} \frac{h_{\kappa}}{p_{\kappa}^{2}}\|\mathbf{w}\|_{0, \partial \kappa}^{2} \lesssim \frac{1}{C_{\eta}}\|\mathbf{w}\|_{0, \Omega}^{2},
$$


where the hidden constants depends on $\mathcal{D}_{*}$.

We endow the space $\widetilde{\mathbf{V}}_{h p}=\mathbf{V}_{h p} \oplus \mathbf{H}_{0, \Gamma_{D}}^{1}(\Omega)$ with the following DG norm

$$
\|\mathbf{v}\|_{\mathrm{DG}}^{2}=\sum_{\kappa \in \mathcal{T}}\left\|\mathcal{D}^{\frac{1}{2}} \varepsilon(\mathbf{v})\right\|_{0, \kappa}^{2}+\left\|\eta^{\frac{1}{2}} \llbracket \mathbf{v} \rrbracket\right\|_{0, \mathcal{F}_{I} \cup \mathcal{F}_{D}}^{2} \quad \forall \mathbf{v} \in \tilde{\mathbf{V}}_{h p}
$$

and prove the following bound.

Lemma 3.3. For any $\boldsymbol{v} \in \widetilde{\boldsymbol{V}}_{h p}$ it holds

$$
\|\mathcal{R}(\llbracket \boldsymbol{v} \rrbracket)\|_{0, \Omega}^{2} \lesssim \frac{1}{C_{\eta}}\left\|\eta^{\frac{1}{2}} \llbracket \boldsymbol{v} \rrbracket\right\|_{0, \mathcal{F}_{I} \cup \mathcal{F}_{D}}^{2}
$$

where $C_{\eta}$ is the constant appearing in the definition of the penalty function, cf. (9).

Proof. If $\mathbf{v} \in \mathbf{H}_{0, \Gamma_{D}}^{1}(\Omega)$, then $\llbracket \mathbf{v} \rrbracket=\mathbf{0}$ and the estimate is trivial. If $\mathbf{v} \in \mathbf{V}_{h p}$, from the definition of the lifting operator 8 we have

$$
\begin{aligned}
& \|\mathcal{R}(\llbracket \mathbf{v} \rrbracket)\|_{0, \Omega}^{2}=\int_{\Omega} \mathcal{R}(\llbracket \mathbf{v} \rrbracket): \mathcal{R}(\llbracket \mathbf{v} \rrbracket) d \mathbf{x} \\
& =-\int_{\mathcal{F}_{I} \cup \mathcal{F}_{D}}\{\mathcal{R}(\llbracket \mathbf{v} \rrbracket)\}: \llbracket \mathbf{v} \rrbracket d \mathbf{s} \\
& \leq\left(\left\|\eta^{-1 / 2}\{\mathcal{R}(\llbracket \mathbf{v} \rrbracket)\}\right\|_{0, \mathcal{F}_{I} \cup \mathcal{F}_{D}}^{2}\right)^{1 / 2}\left(\left\|\eta^{1 / 2} \llbracket \mathbf{v} \rrbracket\right\|_{0, \mathcal{F}_{I} \cup \mathcal{F}_{D}}^{2}\right)^{1 / 2} \\
& \lesssim\left(\frac{1}{C_{\eta}}\|\mathcal{R}(\llbracket \mathbf{v} \rrbracket)\|_{0, \Omega}^{2}\right)^{1 / 2}\left(\left\|\eta^{1 / 2} \llbracket \mathbf{v} \rrbracket\right\|_{0, \mathcal{F}_{I} \cup \mathcal{F}_{D}}^{2}\right)^{1 / 2},
\end{aligned}
$$

where the last step follows from Lemma 3.2 .

The well-posedness of the DG formulation (6) is established in the following lemma. The proof follows based on employing standard arguments together with Lemma 3.3 .

Lemma 3.4. Assume that $\mathcal{T}$ satisfies Assumption 1a), Then,

$$
\begin{aligned}
\widetilde{\mathcal{B}}(\boldsymbol{v}, \boldsymbol{v}) & \gtrsim\|\boldsymbol{v}\|_{D G}^{2}, \\
\widetilde{\mathcal{B}}(\boldsymbol{v}, \boldsymbol{w}) & \lesssim\|\boldsymbol{v}\|_{D G}\|\boldsymbol{w}\|_{D G}
\end{aligned}
$$

for all $\boldsymbol{v}, \boldsymbol{w} \in \widetilde{\boldsymbol{V}}_{h p}$. The first bound holds provided that the constant $C_{\eta}$ appearing in definition (9) of the stabilization function is chosen sufficiently large. 


\subsection{Stability of the semidiscrete formulation}

The aim of this section is to show that the semidiscrete approximation depends continuously on the data. The stability result of the semidiscrete DG formulation (6) in the energy norm

$$
\left\|\mathbf{u}_{h}(t)\right\|_{\mathrm{E}}^{2}=\left\|\rho^{\frac{1}{2}} \dot{\mathbf{u}}_{h}(t)\right\|_{0, \Omega}^{2}+\left\|\mathbf{u}_{h}(t)\right\|_{\mathrm{DG}}^{2} \quad \forall t \in(0, T] .
$$

is established in the following result.

Proposition 3.1. Let $\mathbf{f} \in L^{2}\left((0, T] ; \boldsymbol{L}^{2}(\Omega)\right)$ and let $\mathbf{u}_{h} \in \mathcal{C}^{2}\left((0, T] ; \boldsymbol{V}_{h p}\right)$ be the approximate solution of (6) obtained with the stability constant $C_{\eta}$ defined in (9) chosen sufficiently large, so that Lemma 3.4 holds. Then,

$$
\left\|\mathbf{u}_{h}(t)\right\|_{E} \lesssim\left\|\mathbf{u}_{h}^{0}\right\|_{E}+\int_{0}^{t}\|\mathbf{f}(\tau)\|_{0, \Omega} d \tau, \quad 0<t \leq T .
$$

Before proving Proposition 3.1, we recall the integration by parts formula

$$
\int_{0}^{t}(\mathbf{w}, \dot{\mathbf{v}})_{*} d \tau=(\mathbf{w}(t), \mathbf{v}(t))_{*}-(\mathbf{w}(0), \mathbf{v}(0))_{*}-\int_{0}^{t}(\dot{\mathbf{w}}, \mathbf{v})_{*} d \tau
$$

that holds for $\mathbf{w}, \mathbf{v}$ regular enough and for any scalar product $(\cdot, \cdot \cdot)_{*}$

Proof. We take $\mathbf{v}=\dot{\mathbf{u}}_{h} \in \mathbf{V}_{h p}$ in the variational formulation (6) and integrate it in time between 0 and $t$ getting

$$
\begin{aligned}
\left\|\mathbf{u}_{h}\right\|_{\mathrm{E}}^{2}+2 \int_{\Omega} \mathcal{R}\left(\llbracket \mathbf{u}_{h} \rrbracket\right): \boldsymbol{\sigma}\left(\mathbf{u}_{h}\right) d \mathbf{x}= & \left\|\mathbf{u}_{h}^{0}\right\|_{\mathrm{E}}^{2} \\
& +2 \int_{\Omega} \mathcal{R}\left(\llbracket \mathbf{u}_{h}^{0} \rrbracket\right): \boldsymbol{\sigma}\left(\mathbf{u}_{h}^{0}\right) d \mathbf{x}+2 \int_{0}^{t}\left(\int_{\Omega} \mathbf{f} \cdot \dot{\mathbf{u}}_{h} d \mathbf{x}\right) d \tau .
\end{aligned}
$$

From Lemma 3.2 we have

$$
2\left|\int_{\Omega} \mathcal{R}\left(\llbracket \mathbf{u}_{h} \rrbracket\right): \boldsymbol{\sigma}\left(\mathbf{u}_{h}\right) d \mathbf{x}\right| \lesssim \frac{1}{\sqrt{C_{\eta}}}\left\|\eta^{\frac{1}{2}} \llbracket \mathbf{u}_{h} \rrbracket\right\|_{0, \mathcal{F}_{I} \cup \mathcal{F}_{D}}\left\|\boldsymbol{\sigma}\left(\mathbf{u}_{h}\right)\right\|_{0, \Omega},
$$

from which it follows

$$
\left\|\mathbf{u}_{h}\right\|_{\mathrm{E}}^{2}+2 \int_{\Omega} \mathcal{R}\left(\llbracket \mathbf{u}_{h} \rrbracket\right): \boldsymbol{\sigma}\left(\mathbf{u}_{h}\right) d \mathbf{x} \gtrsim\left\|\mathbf{u}_{h}\right\|_{\mathrm{E}}^{2},
$$

based on employing the arithmetic-geometric inequality and choosing $C_{\eta}$ large enough. Moreover, from Lemma 3.2 it also follows

$$
2\left|\int_{\Omega} \mathcal{R}\left(\llbracket \mathbf{u}_{h} \rrbracket^{0}\right): \boldsymbol{\sigma}\left(\mathbf{u}_{h}^{0}\right) d \mathbf{x}\right| \lesssim \frac{1}{\sqrt{C_{\eta}}}\left\|\eta^{\frac{1}{2}} \llbracket \mathbf{u}_{h}^{0} \rrbracket\right\|_{0, \mathcal{F}_{I} \cup \mathcal{F}_{D}}\left\|\boldsymbol{\sigma}\left(\mathbf{u}_{h}^{0}\right)\right\|_{0, \Omega} \lesssim \frac{1}{\sqrt{C_{\eta}}}\left\|\mathbf{u}_{h}^{0}\right\|_{\mathrm{E}}^{2} .
$$


Therefore, substituting the latter inequalities in 16 and applying the Cauchy-Schwarz inequality to the last term on the right hand side we obtain

$$
\left\|\mathbf{u}_{h}\right\|_{\mathrm{E}}^{2} \lesssim\left\|\mathbf{u}_{h}^{0}\right\|_{\mathrm{E}}^{2}+2 \int_{0}^{t}\left\|\mathbf{u}_{h}\right\|_{\mathrm{E}}\|\mathbf{f}\|_{0, \Omega} d \tau
$$

The thesis follows by employing the Gronwall's lemma [54.

\subsection{A-priori error bounds in the energy norm}

In order to derive a-priori error bounds for the semidiscrete scheme, we define the extension operators $\mathcal{E}: \mathcal{H}^{s}(\Omega) \rightarrow \mathcal{H}^{s}\left(\mathbb{R}^{d \times d}\right), s \in \mathbb{N}_{0}$, such that $\left.\mathcal{E} \tau\right|_{\Omega}=\boldsymbol{\tau}$ and $\varepsilon: \boldsymbol{H}^{s}(\Omega) \rightarrow \boldsymbol{H}^{s}\left(\mathbb{R}^{d}\right)$, $s \in \mathbb{N}_{0}$, such that $\left.\varepsilon \mathbf{v}\right|_{\Omega}=\mathbf{v}$, cf. [55], and recall the following approximation estimates, which are the tensorial and vectorial counterpart, respectively, of the analogous ones shown in [22].

Lemma 3.5. Assume that Assumption 1 holds. Let $\left.\mathbf{v}\right|_{\kappa} \in \mathbf{H}^{m_{\kappa}}(\kappa)$ and $\left.\boldsymbol{\tau}\right|_{\kappa} \in \mathcal{H}^{m_{\kappa}}(\kappa), m_{\kappa}>d / 2$, such that $\left.\varepsilon \mathbf{v}\right|_{\mathcal{K}} \in \mathbf{H}^{m_{k}}(\mathcal{K})$ and $\left.\mathcal{E} \boldsymbol{\tau}\right|_{\mathcal{K}} \in \mathcal{H}^{m_{k}}(\mathcal{K})$, for each $\kappa \in \mathcal{T}$, where $\kappa \subset \mathcal{K}, \mathcal{K} \in \mathcal{T}^{\sharp}$. Then, there exists projection operators $\boldsymbol{\pi}: \mathbf{L}^{2}(\Omega) \rightarrow \boldsymbol{V}_{h p}$ and $\boldsymbol{\Pi}: \mathcal{L}^{2}(\Omega) \longrightarrow \mathcal{V}_{h p}$ such that

$$
\begin{array}{rlrl}
\|\mathbf{v}-\boldsymbol{\pi} \mathbf{v}\|_{\mathbf{H}^{q}(\kappa)} & \lesssim \frac{h_{\kappa}^{s_{\kappa}-q}}{p_{\kappa}^{m_{\kappa}-q}}\|\varepsilon \mathbf{v}\|_{\mathbf{H}^{m_{\kappa}}(\mathcal{K})} & & 0 \leq q \leq m_{\kappa} \\
\|\mathbf{v}-\boldsymbol{\pi} \mathbf{v}\|_{\mathbf{L}^{2}(\partial \kappa)} & \lesssim \frac{h_{\kappa}^{s_{\kappa}-1 / 2}}{p_{\kappa}^{m_{\kappa}-1 / 2}}\|\boldsymbol{\varepsilon} \mathbf{v}\|_{\mathbf{H}^{m_{\kappa}}(\mathcal{K})}, & \\
\|\boldsymbol{\tau}-\boldsymbol{\Pi} \boldsymbol{\tau}\|_{\mathcal{H}^{q}(\kappa)} & \lesssim \frac{h_{\kappa}^{s_{\kappa}-q}}{p_{\kappa}^{m_{\kappa}-q}}\|\mathcal{E} \boldsymbol{\tau}\|_{\mathcal{H}^{m_{\kappa}}(\mathcal{K})} & & 0 \leq q \leq m_{\kappa} \\
\|\boldsymbol{\tau}-\boldsymbol{\Pi} \boldsymbol{\tau}\|_{\mathcal{L}^{2}(\partial \kappa)} & \lesssim \frac{h_{\kappa}^{s_{\kappa}-1 / 2}}{p_{\kappa}^{m_{\kappa}-1 / 2}}\|\mathcal{E} \boldsymbol{\tau}\|_{\mathcal{H}^{m_{\kappa}}(\mathcal{K})}, &
\end{array}
$$

with $s_{\kappa}=\min \left\{p_{\kappa}+1, m_{\kappa}\right\}$. The hidden constants depend on the material properties and the shape regularity constant of the covering $\mathcal{T}^{\sharp}$, cf. Assumption 1, but are independent of the discretization parameters, as well as the number of faces of $\kappa$ and the relative measure of the faces of $\kappa$ compared to the diameter $h_{\kappa}$.

From Lemma 3.5. we obtain the following bounds

$$
\begin{gathered}
\|\mathbf{u}-\boldsymbol{\pi} \mathbf{u}\|_{\mathrm{DG}}^{2} \lesssim \sum_{\kappa \in \mathcal{T}} \frac{h_{\kappa}^{2\left(s_{\kappa}-1\right)}}{p_{\kappa}^{2\left(m_{\kappa}-3 / 2\right)}}\|\varepsilon \mathbf{u}\|_{\mathbf{H}^{m_{k}}(\mathcal{K})}^{2}, \\
\|\mathbf{u}-\boldsymbol{\pi} \mathbf{u}\|_{\mathrm{E}}^{2} \lesssim \sum_{\kappa \in \mathcal{T}} \frac{h_{\kappa}^{2\left(s_{\kappa}-1\right)}}{p_{\kappa}^{2\left(m_{\kappa}-3 / 2\right)}}\left(\|\varepsilon \mathbf{u}\|_{\mathbf{H}^{m_{k}(\mathcal{K})}}^{2}+\frac{h_{\kappa}^{2}}{p_{\kappa}^{3}}\|\varepsilon \dot{\mathbf{u}}\|_{\mathbf{H}^{m_{k}(\mathcal{K})}}^{2}\right),
\end{gathered}
$$

that will be needed in the following analysis. 
Since formulation (6) is not strongly-consistent, we next deal with the consistency error. It is easy to see that the exact solution $\mathbf{u}$ of problem (3) satisfies

$$
\int_{\Omega} \rho \ddot{\mathbf{u}} \cdot \mathbf{v}_{h} d \mathbf{x}+\mathcal{B}\left(\mathbf{u}, \mathbf{v}_{h}\right)=\int_{\Omega} \mathbf{f} \cdot \mathbf{v}_{h} d \mathbf{x} \quad \forall \mathbf{v}_{h} \in \mathbf{V}_{h p}
$$

cf. (4). Defining the residual $\mathcal{R}_{h}(\cdot, \cdot): \widetilde{\mathbf{V}}_{h p} \times \mathbf{V}_{h p} \rightarrow \mathbb{R}$ as

$$
\mathcal{R}_{h}\left(\mathbf{w}, \mathbf{v}_{h}\right)=\mathcal{B}\left(\mathbf{w}, \mathbf{v}_{h}\right)-\widetilde{\mathcal{B}}\left(\mathbf{w}, \mathbf{v}_{h}\right) \quad \forall \mathbf{w} \in \widetilde{\mathbf{V}}_{h p}, \forall \mathbf{v}_{h} \in \mathbf{V}_{h p}
$$

from (6), we get the following error equation

$$
\int_{\Omega} \rho\left(\ddot{\mathbf{u}}-\ddot{\mathbf{u}}_{h}\right) \cdot \mathbf{v}_{h} d \mathbf{x}+\widetilde{\mathcal{B}}\left(\mathbf{u}-\mathbf{u}_{h}, \mathbf{v}_{h}\right)+\mathcal{R}_{h}\left(\mathbf{u}-\mathbf{u}_{h}, \mathbf{v}_{h}\right)=0 \quad \forall \mathbf{v}_{h} \in \mathbf{V}_{h p}
$$

where we have also used that $\mathcal{R}_{h}\left(\mathbf{w}_{h}, \mathbf{v}_{h}\right)=0$ whenever $\mathbf{w}_{h} \in \mathbf{V}_{h p}$. We also have the following

Lemma 3.6. For any $\boldsymbol{\psi}$ such that $\left.\boldsymbol{\sigma}(\boldsymbol{\psi})\right|_{\kappa} \in \mathcal{H}^{m_{k}}(\kappa)$ and $\left.\mathcal{E} \boldsymbol{\sigma}(\boldsymbol{\psi})\right|_{\mathcal{K}} \in \mathcal{H}^{m_{k}}(\mathcal{K}), m_{k} \geq 1$, for each $\kappa \in \mathcal{T}$, where $\kappa \subset \mathcal{K}, \mathcal{K} \in \mathcal{T}^{\sharp}$, then

$$
\left|\mathcal{R}_{h}\left(\boldsymbol{\psi}, \boldsymbol{v}_{h}\right)\right| \lesssim \mathcal{I}(\boldsymbol{\psi})\left\|\eta^{\frac{1}{2}} \llbracket \boldsymbol{v}_{h} \rrbracket\right\|_{0, \mathcal{F}_{I} \cup \mathcal{F}_{D}} \quad \forall \boldsymbol{v}_{h} \in \boldsymbol{V}_{h p}
$$

where

$$
\mathcal{I}^{2}(\boldsymbol{\psi})=\sum_{\kappa \in \mathcal{T}} \frac{h_{\kappa}^{2 s_{\kappa}}}{p_{\kappa}^{2 m_{\kappa}}}\|\mathcal{E} \boldsymbol{\sigma}(\boldsymbol{\psi})\|_{\mathcal{H}^{m_{\kappa}}(\mathcal{K})}^{2}
$$

with $s_{\kappa}=\min \left\{p_{\kappa}+1, m_{\kappa}\right\}$.

Proof. From the definition of the lifting operator $\mathcal{R}$ in $(8)$ and denoting by $\Pi_{0}: \mathcal{L}^{2}(\Omega) \longrightarrow \mathcal{V}_{h p}$ the $L^{2}$-orthogonal projection onto $\mathcal{V}_{h p}$, we can write the residual $\mathcal{R}_{h}(\cdot, \cdot)$ as

$$
\begin{aligned}
\mathcal{R}_{h}\left(\boldsymbol{\psi}, \mathbf{v}_{h}\right) & =-\int_{\mathcal{F}_{I} \cup \mathcal{F}_{D}}\{\boldsymbol{\sigma}(\boldsymbol{\psi})\}: \llbracket \mathbf{v}_{h} \rrbracket d \mathbf{s}-\int_{\Omega} \mathcal{R}\left(\llbracket \mathbf{v}_{h} \rrbracket\right): \boldsymbol{\sigma}(\boldsymbol{\psi}) d \mathbf{x} \\
& =-\int_{\mathcal{F}_{I} \cup \mathcal{F}_{D}}\{\boldsymbol{\sigma}(\boldsymbol{\psi})\}: \llbracket \mathbf{v}_{h} \rrbracket d \mathbf{s}-\int_{\Omega} \mathcal{R}\left(\llbracket \mathbf{v}_{h} \rrbracket\right): \boldsymbol{\Pi}_{0}(\boldsymbol{\sigma}(\boldsymbol{\psi})) d \mathbf{x} \\
& =-\int_{\mathcal{F}_{I} \cup \mathcal{F}_{D}}\left\{\boldsymbol{\sigma}(\boldsymbol{\psi})-\Pi_{0}(\boldsymbol{\sigma}(\boldsymbol{\psi}))\right\}: \llbracket \mathbf{v}_{h} \rrbracket d \mathbf{s} .
\end{aligned}
$$

From the Cauchy-Schwarz inequality we have

$$
\left|\mathcal{R}_{h}\left(\boldsymbol{\psi}, \mathbf{v}_{h}\right)\right| \leq\left\|\eta^{\frac{1}{2}} \llbracket \mathbf{v}_{h} \rrbracket\right\|_{0, \mathcal{F}_{I} \cup \mathcal{F}_{D}}\left\|\eta^{-\frac{1}{2}}\left\{\boldsymbol{\sigma}(\boldsymbol{\psi})-\boldsymbol{\Pi}_{0}(\boldsymbol{\sigma}(\boldsymbol{\psi}))\right\}\right\|_{0, \mathcal{F}_{I} \cup \mathcal{F}_{D}},
$$

where $\eta$ is the penalty parameter function defined ad in $(9)$. By adding and subtracting $\{\boldsymbol{\Pi} \boldsymbol{\sigma}(\boldsymbol{\psi})\}$, 
where $\boldsymbol{\Pi}$ is defined as in Lemma 3.5, we obtain

$$
\begin{aligned}
& \left|\mathcal{R}_{h}\left(\boldsymbol{\psi}, \mathbf{v}_{h}\right)\right| \leq\left(\left\|\eta^{-\frac{1}{2}}\{\boldsymbol{\sigma}(\boldsymbol{\psi})-\boldsymbol{\Pi}(\boldsymbol{\sigma}(\boldsymbol{\psi}))\}\right\|_{0, \mathcal{F}_{I} \cup \mathcal{F}_{D}}^{2}+\left\|\eta^{-\frac{1}{2}}\left\{\boldsymbol{\Pi}(\boldsymbol{\sigma}(\boldsymbol{\psi}))-\boldsymbol{\Pi}_{0}(\boldsymbol{\sigma}(\boldsymbol{\psi}))\right\}\right\|_{0, \mathcal{F}_{I} \cup \mathcal{F}_{D}}^{2}\right)^{1 / 2} \| \eta^{\frac{1}{2} \llbracket \mathbf{v}_{h} \rrbracket \|_{0, \mathcal{F}_{I} \cup \mathcal{F}_{D}}} \\
& =\left(T_{1}+T_{2}\right)^{1 / 2}\left\|\eta^{\frac{1}{2}} \llbracket \mathbf{v}_{h} \rrbracket\right\|_{0, \mathcal{F}_{I} \cup \mathcal{F}_{D}} .
\end{aligned}
$$

The term $T_{1}$ can be bounded based on employing the interpolation estimates of Lemma 3.5

$$
T_{1} \lesssim \sum_{\kappa \in \mathcal{T}}\left\|\eta^{-1 / 2}(\boldsymbol{\sigma}(\boldsymbol{\psi})-\boldsymbol{\Pi}(\boldsymbol{\sigma}(\boldsymbol{\psi})))\right\|_{\mathcal{L}^{2}(\partial \kappa)}^{2} \lesssim \sum_{\kappa \in \mathcal{T}} \frac{h_{\kappa}^{2 s_{\kappa}}}{p_{\kappa}^{2 m_{\kappa}+1}}\|\mathcal{E} \boldsymbol{\sigma}(\boldsymbol{\psi})\|_{\mathcal{H}^{m_{\kappa}}(\mathcal{K})}^{2}
$$

For $T_{2}$, from Lemma 3.2 the definition of the $L^{2}$-projection operator together with its continuity, and the interpolation estimates of Lemma 3.5 we have

$$
\begin{aligned}
T_{2} & \lesssim \sum_{\kappa \in \mathcal{T}}\left\|\eta^{-1 / 2}\left\{\boldsymbol{\Pi}(\boldsymbol{\sigma}(\boldsymbol{\psi}))-\boldsymbol{\Pi}_{0}(\boldsymbol{\sigma}(\boldsymbol{\psi}))\right\}\right\|_{0, \partial \kappa}^{2} \lesssim \frac{1}{C_{\eta}} \sum_{\kappa \in \mathcal{T}}\left\|\boldsymbol{\Pi}(\boldsymbol{\sigma}(\boldsymbol{\psi}))-\boldsymbol{\Pi}_{0}(\boldsymbol{\sigma}(\boldsymbol{\psi}))\right\|_{0, \kappa}^{2} \\
& =\frac{1}{C_{\eta}} \sum_{\kappa \in \mathcal{T}}\left\|\boldsymbol{\Pi}_{0}(\boldsymbol{\Pi}(\boldsymbol{\sigma}(\boldsymbol{\psi}))-\boldsymbol{\sigma}(\boldsymbol{\psi}))\right\|_{0, \kappa}^{2} \leq \frac{1}{C_{\eta}} \sum_{\kappa \in \mathcal{T}}\|\boldsymbol{\Pi}(\boldsymbol{\sigma}(\boldsymbol{\psi}))-\boldsymbol{\sigma}(\boldsymbol{\psi})\|_{0, \kappa}^{2} \\
& \lesssim \frac{1}{C_{\eta}} \sum_{\kappa \in \mathcal{T}} \frac{h_{\kappa}^{2 s_{\kappa}}}{p_{\kappa}^{2 m_{\kappa}}}\|\mathcal{E} \boldsymbol{\sigma}(\boldsymbol{\psi})\|_{\mathcal{H}^{m_{\kappa}}(\mathcal{K})} .
\end{aligned}
$$

Summing up the two contributions we get

$$
\begin{aligned}
\left|\mathcal{R}_{h}\left(\boldsymbol{\psi}, \mathbf{v}_{h}\right)\right| & \lesssim\left(\sum_{\kappa \in \mathcal{T}} \frac{h_{\kappa}^{2 s_{\kappa}}}{p_{\kappa}^{2 m_{\kappa}+1}}\|\mathcal{E} \boldsymbol{\sigma}(\boldsymbol{\psi})\|_{\mathcal{H}^{m_{\kappa}}(\mathcal{K})}^{2}+\sum_{\kappa \in \mathcal{T}} \frac{h_{\kappa}^{2 s_{\kappa}}}{p_{\kappa}^{2 m_{\kappa}}}\|\mathcal{E} \boldsymbol{\sigma}(\boldsymbol{\psi})\|_{\mathcal{H}^{m_{\kappa}}(\mathcal{K})}\right)^{1 / 2}\left\|\eta^{\frac{1}{2}} \llbracket \mathbf{v}_{h} \rrbracket\right\|_{0, \mathcal{F}_{I} \cup \mathcal{F}_{D}} \\
& \lesssim\left(\sum_{\kappa \in \mathcal{T}} \frac{h_{\kappa}^{2 s_{\kappa}}}{p_{\kappa}^{2 m_{\kappa}}}\|\mathcal{E} \boldsymbol{\sigma}(\boldsymbol{\psi})\|_{\mathcal{H}^{m_{\kappa}}(\mathcal{K})}^{2}\right)^{1 / 2}\left\|\eta^{\frac{1}{2}} \llbracket \mathbf{v}_{h} \rrbracket\right\|_{0, \mathcal{F}_{I} \cup \mathcal{F}_{D}}
\end{aligned}
$$

135 and the proof is complete.

Remark 1. If the mesh $\mathcal{T}$ is quasi uniform, $p_{\kappa}=p$ and $m_{\kappa}=m \forall \kappa \in \mathcal{T}$, then

$$
\mathcal{I}^{2}(\boldsymbol{\psi})=\frac{h^{2 s}}{p^{2 m}} \sum_{\kappa \in \mathcal{T}}\|\mathcal{E} \boldsymbol{\sigma}(\boldsymbol{\psi})\|_{\mathcal{H}^{m_{\kappa}(\mathcal{K})}}^{2} \lesssim \frac{h^{2 s}}{p^{2 m}}\|\boldsymbol{\sigma}(\boldsymbol{\psi})\|_{\mathcal{H}^{m}(\Omega)}^{2}
$$

with $s=\min \{p+1, m\}$, provided that $\boldsymbol{\psi}$ is regular enough. We notice that the last step follows based on employing the finite covering assumption (10) and the continuity of the extension operator, cf. also [20, Remark 5.4].

We have now all the technical tools and can state the main result for the error analysis. 
Theorem 3.2. Assume that Assumption 1 holds and that the exact solution $\mathbf{u}$ of (1) is sufficiently regular. For any time $t \in[0, T]$, let $\mathbf{u}_{h} \in \boldsymbol{V}_{h p}$ be the $D G$ solution of problem (4) obtained with a penalty parameter $C_{\eta}$ appearing in (9) sufficiently large. Then, the following bound holds

$$
\begin{aligned}
\sup _{0<t \leq T}\left\|\mathbf{u}(t)-\mathbf{u}_{h}(t)\right\|_{E}^{2} \lesssim & \sum_{\kappa \in \mathcal{T}} \frac{h_{\kappa}^{2\left(s_{k}-1\right)}}{p_{\kappa}^{2\left(m_{k}-3 / 2\right)}}\left(\|\varepsilon \mathbf{u}\|_{\mathbf{H}^{m_{k}(\mathcal{K})}}^{2}+\frac{h_{\kappa}^{2}}{p_{\kappa}^{3}}\|\varepsilon \dot{\mathbf{u}}\|_{\mathbf{H}^{m_{k}(\mathcal{K})}}^{2}+\frac{h_{\kappa}^{2}}{p_{\kappa}^{3}}\|\mathcal{E} \boldsymbol{\sigma}(\boldsymbol{u})\|_{\mathcal{H}^{m_{k}(\mathcal{K})}}^{2}\right) \\
& +\frac{h_{\kappa}^{2\left(s_{\kappa}-1\right)}}{p_{\kappa}^{2\left(m_{k}-3 / 2\right)}} \int_{0}^{t}\left(\|\varepsilon \dot{\mathbf{u}}\|_{\mathbf{H}^{m_{k}(\mathcal{K})}}^{2}+\frac{h_{\kappa}^{2}}{p_{\kappa}^{3}}\|\varepsilon \ddot{\mathbf{u}}\|_{\mathbf{H}^{m_{k}(\mathcal{K})}}^{2}+\frac{h_{\kappa}^{2}}{p_{\kappa}^{3}}\|\mathcal{E} \boldsymbol{\sigma}(\dot{\boldsymbol{u}})\|_{\mathcal{H}^{m_{k}(\mathcal{K})}}^{2}\right) d \tau
\end{aligned}
$$

with $s_{\kappa}=\min \left(p_{\kappa}+1, m_{k}\right)$ for all $\kappa \in \mathcal{T}$.

Proof. Let $\boldsymbol{\pi}$ be defined as in Lemma 3.5 and let $\mathbf{e}_{h}=\mathbf{u}_{h}-\boldsymbol{\pi} \mathbf{u}$. We write the error equation 25 for $\mathbf{v}=\dot{\mathbf{e}}_{h}$, obtaining

$$
\int_{\Omega} \rho\left(\ddot{\mathbf{u}}-\ddot{\mathbf{u}}_{h}\right) \cdot \dot{\mathbf{e}}_{h} d \mathbf{x}+\widetilde{\mathcal{B}}\left(\mathbf{u}-\mathbf{u}_{h}, \dot{\mathbf{e}}_{h}\right)+\mathcal{R}_{h}\left(\mathbf{u}-\mathbf{u}_{h}, \dot{\mathbf{e}}_{h}\right)=0
$$

Writing $\mathbf{u}-\mathbf{u}_{h}=\mathbf{e}_{I}-\mathbf{e}_{h}$, with $\mathbf{e}_{I}=\mathbf{u}-\boldsymbol{\pi} \mathbf{u}$, we have

$$
\int_{\Omega} \rho \ddot{\mathbf{e}}_{h} \cdot \dot{\mathbf{e}}_{h} d \mathbf{x}+\widetilde{\mathcal{B}}\left(\mathbf{e}_{h}, \dot{\mathbf{e}}_{h}\right)=\int_{\Omega} \rho \ddot{\mathbf{e}}_{I} \cdot \dot{\mathbf{e}}_{h} d \mathbf{x}+\widetilde{\mathcal{B}}\left(\mathbf{e}_{I}, \dot{\mathbf{e}}_{h}\right)+\mathcal{R}_{h}\left(\mathbf{e}_{I}, \dot{\mathbf{e}}_{h}\right)
$$

where we have also used that $\mathcal{R}_{h}\left(\mathbf{e}_{h}, \dot{\mathbf{e}}_{h}\right)=0$ since $\mathbf{e}_{h}, \dot{\mathbf{e}}_{h} \in \mathbf{V}_{h p}$. Using the definition of the energy norm 13 , the above equation is equivalent to

$$
\frac{1}{2} \frac{d}{d t}\left(\left\|\mathbf{e}_{h}\right\|_{\mathrm{E}}^{2}+2 \int_{\Omega} \mathcal{R}\left(\llbracket \mathbf{e}_{h} \rrbracket\right): \boldsymbol{\sigma}\left(\mathbf{e}_{h}\right) d \mathbf{x}\right)=\int_{\Omega} \rho \ddot{\mathbf{e}}_{I} \cdot \dot{\mathbf{e}}_{h} d \mathbf{x}+\widetilde{\mathcal{B}}\left(\mathbf{e}_{I}, \dot{\mathbf{e}}_{h}\right)+\mathcal{R}_{h}\left(\mathbf{e}_{I}, \dot{\mathbf{e}}_{h}\right)
$$

Integrating in time between 0 and $t$, exploiting that $\mathbf{e}_{h}(0)=\mathbf{0}$, and reasoning as in the proof of Proposition 3.1 yield

$$
\left\|\mathbf{e}_{h}\right\|_{\mathrm{E}}^{2}+2 \int_{\Omega} \mathcal{R}\left(\llbracket \mathbf{e}_{h} \rrbracket\right): \boldsymbol{\sigma}\left(\mathbf{e}_{h}\right) d \mathbf{x} \gtrsim\left\|\mathbf{e}_{h}\right\|_{\mathrm{E}}^{2}
$$

provided the penalty parameter $C_{\eta}$ appearing in the definition $\sqrt{9}$ is chosen sufficiently large. Therefore, we get

$$
\begin{aligned}
\left\|\mathbf{e}_{h}\right\|_{\mathrm{E}}^{2} & \lesssim \int_{0}^{t} \int_{\Omega} \rho \ddot{\mathbf{e}}_{I} \cdot \dot{\mathbf{e}}_{h} d \mathbf{x} d \tau+\int_{0}^{t} \widetilde{\mathcal{B}}\left(\mathbf{e}_{I}, \dot{\mathbf{e}}_{h}\right) d \tau+\int_{0}^{t} \mathcal{R}_{h}\left(\mathbf{e}_{I}, \dot{\mathbf{e}}_{h}\right) d \tau \\
& =\int_{0}^{t} \int_{\Omega} \rho \ddot{\mathbf{e}}_{I} \cdot \dot{\mathbf{e}}_{h} d \mathbf{x} d \tau+\widetilde{\mathcal{B}}\left(\mathbf{e}_{I}, \mathbf{e}_{h}\right)-\int_{0}^{t} \widetilde{\mathcal{B}}\left(\dot{\mathbf{e}}_{I}, \mathbf{e}_{h}\right) d \tau-\mathcal{R}_{h}\left(\mathbf{e}_{I}, \mathbf{e}_{h}\right)+\int_{0}^{t} \mathcal{R}_{h}\left(\dot{\mathbf{e}}_{I}, \mathbf{e}_{h}\right) d \tau,
\end{aligned}
$$

where in the second step we have used the integration by parts formula 15 for the second and 
third terms on the right hand side together with $\mathbf{e}_{h}(0)=\mathbf{0}$. From the Cauchy-Schwarz inequality for first term on the right hand side we obtain

$$
\begin{aligned}
\left\|\mathbf{e}_{h}\right\|_{\mathrm{E}}^{2} \lesssim \int_{0}^{t}\left\|\rho^{\frac{1}{2}} \ddot{\mathbf{e}}_{I}\right\|_{\mathbf{L}^{2}(\Omega)}\left\|\rho^{\frac{1}{2}} \dot{\mathbf{e}}_{h}\right\|_{\mathbf{L}^{2}(\Omega)} d \tau & +\widetilde{\mathcal{B}}\left(\mathbf{e}_{I}, \mathbf{e}_{h}\right) \\
& +\int_{0}^{t} \widetilde{\mathcal{B}}\left(\dot{\mathbf{e}}_{I}, \mathbf{e}_{h}\right) d \tau-\mathcal{R}_{h}\left(\mathbf{e}_{I}, \mathbf{e}_{h}\right)+\int_{0}^{t} \mathcal{R}_{h}\left(\dot{\mathbf{e}}_{I}, \mathbf{e}_{h}\right) d \tau .
\end{aligned}
$$

We next observe that, from the definition of the residual $\mathcal{R}_{h}\left(\mathbf{e}_{I}, \mathbf{e}_{h}\right)=\mathcal{R}_{h}\left(\mathbf{u}, \mathbf{e}_{h}\right)$, and the above equation becomes

$$
\begin{aligned}
\left\|\mathbf{e}_{h}\right\|_{\mathrm{E}}^{2} \lesssim \int_{0}^{t}\left\|\rho^{\frac{1}{2}} \ddot{\mathbf{e}}_{I}\right\|_{\mathbf{L}^{2}(\Omega)}\left\|\rho^{\frac{1}{2}} \dot{\mathbf{e}}_{h}\right\|_{\mathbf{L}^{2}(\Omega)} d \tau & +\widetilde{\mathcal{B}}\left(\mathbf{e}_{I}, \mathbf{e}_{h}\right) \\
& +\int_{0}^{t} \widetilde{\mathcal{B}}\left(\dot{\mathbf{e}}_{I}, \mathbf{e}_{h}\right) d \tau-\mathcal{R}_{h}\left(\mathbf{u}, \mathbf{e}_{h}\right)+\int_{0}^{t} \mathcal{R}_{h}\left(\dot{\mathbf{u}}, \mathbf{e}_{h}\right) d \tau .
\end{aligned}
$$

Using Lemma 3.4 the definition of the energy norm 13, and Lemma 3.6 we obtain

$$
\left\|\mathbf{e}_{h}\right\|_{\mathrm{E}}^{2} \lesssim\left\|\mathbf{e}_{I}\right\|_{\mathrm{E}}\left\|\mathbf{e}_{h}\right\|_{\mathrm{E}}+\int_{0}^{t}\left\|\dot{\mathbf{e}}_{I}\right\|_{\mathrm{E}}\left\|\mathbf{e}_{h}\right\|_{\mathrm{E}} d \tau+\mathcal{I}(\mathbf{u})\left\|\mathbf{e}_{h}\right\|_{\mathrm{E}}+\int_{0}^{t} \mathcal{I}(\dot{\mathbf{u}})\left\|\mathbf{e}_{h}\right\|_{\mathrm{E}} d \tau
$$

where $\mathcal{I}(\mathbf{u})$ is defined as in 27, cf. Lemma 3.6. Applying the arithmetic-geometric inequality with $\delta>0$ we have

$$
(1-\delta)\left\|\mathbf{e}_{h}\right\|_{\mathrm{E}}^{2} \lesssim \frac{1}{\delta}\left(\left\|\mathbf{e}_{I}\right\|_{\mathrm{E}}^{2}+\mathcal{I}^{2}(\mathbf{u})\right)+\int_{0}^{t}\left(\left\|\dot{\mathbf{e}}_{I}\right\|_{\mathrm{E}}+\mathcal{I}(\dot{\mathbf{u}})\right)\left\|\mathbf{e}_{h}\right\|_{\mathrm{E}} d \tau .
$$

Choosing $\delta$ small enough and applying Gronwall's lemma [54 we get

$$
\left\|\mathbf{e}_{h}\right\|_{\mathrm{E}}^{2} \lesssim\left\|\mathbf{e}_{I}\right\|_{\mathrm{E}}^{2}+\mathcal{I}^{2}(\mathbf{u})+\int_{0}^{t}\left(\left\|\dot{\mathbf{e}}_{I}\right\|_{\mathrm{E}}^{2}+\mathcal{I}^{2}(\dot{\mathbf{u}})\right) d \tau
$$

The proof is completed using $(22)$, the definition of $\mathcal{I}(\mathbf{u})$ and taking the supremum over $t \in$ $(0, T]$. 


\subsection{A-priori error bounds in the $\mathbf{L}^{2}$-norm}

In this section we present a priori error estimates in the $\mathbf{L}^{2}$-norm. In the following, we assume that the grid $\mathcal{T}$ is quasi uniform, $p_{\kappa}=p$ and $m_{\kappa}=m$ for all $\kappa \in \mathcal{T}$. Moreover, we assume that $\Omega$ and $\mathcal{D}$ are sufficiently regular so that $\mathbf{u}, \dot{\mathbf{u}}, \ddot{\mathbf{u}}$ and $\boldsymbol{\sigma}=\mathcal{D} \boldsymbol{\varepsilon}(\mathbf{u})$ posses the following regularity, $\mathbf{u}, \dot{\mathbf{u}}, \ddot{\mathbf{u}} \in \mathbf{H}^{m}(\Omega)$ and $\boldsymbol{\sigma} \in \mathbf{H}^{m}(\Omega)$ for some $m \geq 2$. Since we will make use of a duality argument we also assume that $\Omega$ and $\mathcal{D}$ are sufficiently regular so that, for $\mathbf{g} \in \mathbf{L}^{2}(\Omega)$ the problem

$$
-\nabla \cdot(\mathcal{D} \varepsilon(\boldsymbol{\xi}))=\mathbf{g} \quad \text { in } \Omega, \quad \boldsymbol{\xi}=\mathbf{0} \quad \text { on } \partial \Omega
$$

is well posed and its unique solution $\boldsymbol{\xi}$ satisfies the following elliptic regularity: $\boldsymbol{\xi} \in \mathbf{H}^{2}(\Omega)$, $\boldsymbol{\sigma}(\boldsymbol{\xi})=\mathcal{D} \boldsymbol{\varepsilon}(\boldsymbol{\xi}) \in \mathcal{H}^{1}(\Omega),\|\boldsymbol{\xi}\|_{\mathbf{H}^{2}(\Omega)} \lesssim\|\mathbf{g}\|_{\mathbf{L}^{2}(\Omega)}$ and $\|\boldsymbol{\sigma}(\boldsymbol{\xi})\|_{\mathcal{H}^{1}(\Omega)} \lesssim\|\mathbf{g}\|_{\mathbf{L}^{2}(\Omega)}$. Then, the following bound holds.

Theorem 3.3. Assume that Assumption 1 holds. For any time $t \in[0, T]$, let $\mathbf{u}_{h} \in \boldsymbol{V}_{h p}$ be the $D G$ solution of problem (4) obtained with a penalty parameter $C_{\eta}$ appearing in (9) sufficiently large. Then,

$$
\sup _{0<t \leq T}\left\|\mathbf{u}(t)-\mathbf{u}_{h}(t)\right\|_{L^{2}(\Omega)} \lesssim \frac{h^{s}}{p^{m-1}}\left(\mathcal{N}(\mathbf{u})+\int_{0}^{t} \mathcal{N}(\dot{\mathbf{u}}) d \tau+\frac{h}{p^{2}}\|\boldsymbol{\sigma}(\mathbf{u})\|_{\mathcal{H}^{m}(\Omega)}\right)
$$

with $s=\min (p+1, m)$ and where

$$
\mathcal{N}(\mathbf{u})=\left(\|\mathbf{u}\|_{\mathbf{H}^{m}(\Omega)}^{2}+\frac{h^{2}}{p^{3}}\|\dot{\boldsymbol{u}}\|_{\mathbf{H}^{m}(\Omega)}^{2}+\frac{h^{2}}{p^{3}}\|\boldsymbol{\sigma}(\mathbf{u})\|_{\mathcal{H}^{m}(\Omega)}^{2}\right)^{\frac{1}{2}} .
$$

Proof. We solve (32) with $\mathbf{g}=\mathbf{u}-\mathbf{u}_{h}$ and for any $\boldsymbol{\xi}_{h} \in \mathbf{V}_{h p}$ obtain

$$
\begin{aligned}
\left\|\mathbf{u}-\mathbf{u}_{h}\right\|_{\mathbf{L}^{2}(\Omega)}^{2} & =\mathcal{B}\left(\boldsymbol{\xi}, \mathbf{u}-\mathbf{u}_{h}\right)=\mathcal{B}\left(\boldsymbol{\xi}-\boldsymbol{\xi}_{h}, \mathbf{u}-\mathbf{u}_{h}\right) \\
& =\widetilde{\mathcal{B}}\left(\boldsymbol{\xi}-\boldsymbol{\xi}_{h}, \mathbf{u}-\mathbf{u}_{h}\right)+\mathcal{R}_{h}\left(\boldsymbol{\xi}-\boldsymbol{\xi}_{h}, \mathbf{u}-\mathbf{u}_{h}\right) \\
& \lesssim\left\|\boldsymbol{\xi}-\boldsymbol{\xi}_{h}\right\|_{\mathrm{DG}}\left\|\mathbf{u}-\mathbf{u}_{h}\right\|_{\mathrm{DG}}+\mathcal{R}_{h}\left(\boldsymbol{\xi}-\boldsymbol{\xi}_{h}, \mathbf{u}-\mathbf{u}_{h}\right) .
\end{aligned}
$$

Moreover, thanks to the definition of $\mathcal{R}_{h}$ in $(24)$ and the continuity of $\widetilde{\mathcal{B}}(\cdot, \cdot)$ we have that

$$
\mathcal{R}_{h}\left(\boldsymbol{\xi}-\boldsymbol{\xi}_{h}, \mathbf{u}-\mathbf{u}_{h}\right)=-\mathcal{R}_{h}\left(\boldsymbol{\xi}-\boldsymbol{\xi}_{h}, \mathbf{u}\right)+\mathcal{R}_{h}\left(\boldsymbol{\xi}, \mathbf{u}-\mathbf{u}_{h}\right)
$$

Next, from Lemma 3.6. for any $\boldsymbol{\xi}_{h} \in \mathbf{V}_{h p}$ we have that

$$
\begin{aligned}
\left|\mathcal{R}_{h}\left(\boldsymbol{\psi}, \boldsymbol{\xi}_{h}\right)\right| & \lesssim \mathcal{I}(\boldsymbol{\psi})\left\|\boldsymbol{\xi}_{h}\right\|_{\mathrm{DG}} \\
\left|\mathcal{R}_{h}\left(\boldsymbol{\xi}_{h}, \boldsymbol{\psi}\right)\right| & \lesssim \mathcal{I}(\boldsymbol{\psi})\left\|\boldsymbol{\xi}_{h}\right\|_{\mathrm{DG}}
\end{aligned}
$$


provided that $\boldsymbol{\psi}$ is sufficiently regular. We therefore get from 35

$$
\left\|\mathbf{u}-\mathbf{u}_{h}\right\|_{\mathbf{L}^{2}(\Omega)}^{2} \lesssim\left\|\boldsymbol{\xi}-\boldsymbol{\xi}_{h}\right\|_{\mathrm{DG}}\left\|\mathbf{u}-\mathbf{u}_{h}\right\|_{\mathrm{DG}}+\mathcal{I}(\mathbf{u})\left\|\boldsymbol{\xi}-\boldsymbol{\xi}_{h}\right\|_{\mathrm{DG}}+\mathcal{I}(\boldsymbol{\xi})\left\|\mathbf{u}-\mathbf{u}_{h}\right\|_{\mathrm{DG}}
$$

Now, using estimate 21) (for $m=2$ and $p=1$ ) we have

$$
\|\boldsymbol{\xi}-\boldsymbol{\pi} \boldsymbol{\xi}\|_{\mathrm{DG}} \lesssim \frac{h}{p^{\frac{1}{2}}}\|\boldsymbol{\xi}\|_{\mathbf{H}^{2}(\Omega)} \lesssim \frac{h}{p^{\frac{1}{2}}}\left\|\mathbf{u}-\mathbf{u}_{h}\right\|_{\mathbf{L}^{2}(\Omega)},
$$

while from estimate (28) we have

$$
\begin{aligned}
\mathcal{I}(\mathbf{u}) & \lesssim \frac{h^{s}}{p^{m}}\|\boldsymbol{\sigma}(\mathbf{u})\|_{\mathcal{H}^{m}(\Omega)}, \\
\mathcal{I}(\boldsymbol{\xi}) & \left.\lesssim \frac{h}{p} \| \boldsymbol{\sigma}(\boldsymbol{\xi})\right)\left\|_{\mathcal{H}^{1}(\Omega)} \lesssim \frac{h}{p}\right\| \mathbf{u}-\mathbf{u}_{h} \|_{\mathbf{L}^{2}(\Omega)} .
\end{aligned}
$$

Combining the above bounds and choosing $\boldsymbol{\xi}_{h}=\boldsymbol{\pi} \boldsymbol{\xi}$, where $\boldsymbol{\pi}$ is defined as in Lemma 3.5, we get for any time $t \in(0, T]$

$$
\begin{aligned}
\left\|\mathbf{u}-\mathbf{u}_{h}\right\|_{\mathbf{L}^{2}(\Omega)}^{2} \lesssim & \frac{h}{p^{\frac{1}{2}}}\left\|\mathbf{u}-\mathbf{u}_{h}\right\|_{\mathbf{L}^{2}(\Omega)}\left\|\mathbf{u}-\mathbf{u}_{h}\right\|_{\mathrm{DG}}+\frac{h^{s+1}}{p^{m+1}}\|\boldsymbol{\sigma}(\mathbf{u})\|_{\mathcal{H}^{m}(\Omega)}\left\|\mathbf{u}-\mathbf{u}_{h}\right\|_{\mathbf{L}^{2}(\Omega)} \\
& +\frac{h}{p}\left\|\mathbf{u}-\mathbf{u}_{h}\right\|_{\mathbf{L}^{2}(\Omega)}\left\|\mathbf{u}-\mathbf{u}_{h}\right\|_{\mathrm{DG}} \\
\lesssim & \frac{h}{p^{\frac{1}{2}}}\left(\left\|\mathbf{u}-\mathbf{u}_{h}\right\|_{\mathrm{DG}}+\frac{h^{s}}{p^{m+\frac{1}{2}}}\|\boldsymbol{\sigma}(\mathbf{u})\|_{\mathcal{H}^{m}(\Omega)}\right)\left\|\mathbf{u}-\mathbf{u}_{h}\right\|_{\mathbf{L}^{2}(\Omega)}
\end{aligned}
$$

from which we have

$$
\left\|\mathbf{u}-\mathbf{u}_{h}\right\|_{\mathbf{L}^{2}(\Omega)} \lesssim \frac{h}{p^{\frac{1}{2}}}\left(\left\|\mathbf{u}-\mathbf{u}_{h}\right\|_{\mathrm{DG}}+\frac{h^{s}}{p^{m+\frac{1}{2}}}\|\boldsymbol{\sigma}(\mathbf{u})\|_{\mathcal{H}^{m}(\Omega)}\right)
$$

Finally, applying Theorem 3.2 (for quasi uniform meshes, and $p_{\kappa}=p$ and $m_{\kappa}=m$ for any $\kappa \in \mathcal{T}$ ) we get

$$
\begin{aligned}
\left\|\mathbf{u}-\mathbf{u}_{h}\right\|_{\mathrm{DG}}^{2} \leq\left\|\mathbf{u}-\mathbf{u}_{h}\right\|_{\mathrm{E}}^{2} \lesssim & \frac{h^{2(s-1)}}{p^{2(m-3 / 2)}}\left(\|\mathbf{u}\|_{\mathbf{H}^{m}(\Omega)}^{2}+\frac{h^{2}}{p^{3}}\|\dot{\mathbf{u}}\|_{\mathbf{H}^{m}(\Omega)}^{2}+\frac{h^{2}}{p^{3}}\|\boldsymbol{\sigma}(\mathbf{u})\|_{\mathcal{H}^{m}(\Omega)}^{2}\right) \\
& +\frac{h^{2(s-1)}}{p^{2(m-3 / 2)}} \int_{0}^{t}\left(\|\dot{\mathbf{u}}\|_{\mathbf{H}^{m}(\Omega)}^{2}+\frac{h^{2}}{p^{3}}\|\ddot{\mathbf{u}}\|_{\mathbf{H}^{m}(\Omega)}^{2}+\frac{h^{2}}{p^{3}}\|\boldsymbol{\sigma}(\dot{\mathbf{u}})\|_{\mathcal{H}^{m}(\Omega)}^{2}\right) d \tau,
\end{aligned}
$$

where we have also used the finite-covering assumption (1b) and the continuity of the extension operator $\varepsilon$ and $\mathcal{E}$, cf. [20, Remark 5.4]. Setting

$$
\mathcal{N}^{2}(\mathbf{u})=\|\mathbf{u}\|_{\mathbf{H}^{m}(\Omega)}^{2}+\frac{h^{2}}{p^{3}}\|\dot{\mathbf{u}}\|_{\mathbf{H}^{m}(\Omega)}^{2}+\frac{h^{2}}{p^{3}}\|\boldsymbol{\sigma}(\mathbf{u})\|_{\mathcal{H}^{m}(\Omega)}^{2},
$$


we get

$$
\left\|\mathbf{u}-\mathbf{u}_{h}\right\|_{\mathrm{DG}} \lesssim \frac{h^{s-1}}{p^{m-3 / 2}}\left(\mathcal{N}(\mathbf{u})+\int_{0}^{t} \mathcal{N}(\dot{\mathbf{u}}) d \tau\right) .
$$

Using the above bound in 36 we finally obtain

$$
\begin{aligned}
\left\|\mathbf{u}-\mathbf{u}_{h}\right\|_{\mathbf{L}^{2}(\Omega)} & \lesssim \frac{h}{p^{\frac{1}{2}}}\left(\frac{h^{s-1}}{p^{m-3 / 2}}\left(\mathcal{N}(\mathbf{u})+\int_{0}^{t} \mathcal{N}(\dot{\mathbf{u}}) d \tau\right)+\frac{h^{s}}{p^{m+\frac{1}{2}}}\|\boldsymbol{\sigma}(\mathbf{u})\|_{\mathcal{H}^{m}(\Omega)}\right) \\
& \lesssim \frac{h^{s}}{p^{m-1}}\left(\mathcal{N}(\mathbf{u})+\int_{0}^{t} \mathcal{N}(\dot{\mathbf{u}}) d \tau+\frac{h}{p^{2}}\|\boldsymbol{\sigma}(\mathbf{u})\|_{\mathcal{H}^{m}(\Omega)}\right),
\end{aligned}
$$

and the proof is complete.

\section{Algebraic formulation and time integration}

In this section we present the algebraic formulation and the time marching scheme. We suppose $\Omega$ to be partitioned into $N_{e l}$ disjoint polytopic elements $\kappa_{r}, r=1, \ldots, N_{e l}$, denote by $n_{p_{\kappa}}=$ $\operatorname{dim}\left(\mathbb{P}_{p_{\kappa}}\right)$, and set $N_{d o f}=\sum_{r=1}^{N_{e l}} n_{p_{\kappa}}$ to be the dimension of each component of a function in $\mathbf{V}_{h p}$. We introduce a (modal) basis $\left\{\boldsymbol{\Phi}_{i}^{1}, \ldots, \boldsymbol{\Phi}_{i}^{d}\right\}_{i=1}^{N_{d o f}}, d=2,3$, for the finite element space $\mathbf{V}_{h p}$, where $\boldsymbol{\Phi}_{i}^{s}(\mathbf{x})=\left(0, \ldots, \Phi_{i}^{s}(\mathbf{x}), \ldots, 0\right)^{T}$. By expressing $\mathbf{u}_{h} \in \mathbf{V}_{h p}$ as a linear combination of the basis functions, i.e.,

$$
\mathbf{u}_{h}(\mathbf{x}, t)=\sum_{s=1}^{d} \sum_{j=1}^{N_{\text {dof }}} \boldsymbol{\Phi}_{j}^{s}(\mathbf{x}) U_{j}^{s}(t)
$$

and writing equation (4) for any test function $\boldsymbol{\Phi}_{i}^{s}(\mathbf{x}) \in \mathbf{V}_{h p}, s=1, \ldots, d$, we obtain the following system of second order differential equations

$$
M \ddot{\mathbf{U}}(t)+B \mathbf{U}(t)=\mathbf{F}(t), \quad \forall t \in(0, T)
$$

for the displacement $\mathbf{U}(t)=\left(\mathbf{U}^{1}(t), \ldots, \mathbf{U}^{d}(t)\right)^{T}$. Here, $\mathbf{F}=\left(\mathbf{F}^{1}(t), \ldots, \mathbf{F}^{d}(t)\right)^{T}$ represents the external applied load, $M$ and $B$ are the (symmetric and positive definite) mass and stiffness matrices, respectively. To integrate system [38 in time we apply the leap-frog scheme, which is second-order accurate, explicit and conditionally stable [56]. We subdivide the interval $(0, T]$ into $N_{T}$ subinterval of amplitude $\Delta t=T / N_{T}$ and at every time level $t_{n}=n \Delta t$ we solve the system

$$
M \mathbf{U}\left(t_{n+1}\right)=\left[2 M-\Delta t^{2} B\right] \mathbf{U}\left(t_{n}\right)-M \mathbf{U}\left(t_{n-1}\right)+\Delta t^{2} \mathbf{F}\left(t_{n}\right), \quad \text { for } n=1, \ldots, N_{T}
$$

with

$$
M \mathbf{U}\left(t_{1}\right)=\left[M-\frac{\Delta t^{2}}{2} B\right] \mathbf{U}\left(t_{0}\right)-\Delta t M \dot{\mathbf{U}}\left(t_{0}\right)+\frac{\Delta t^{2}}{2} \mathbf{F}\left(t_{0}\right),
$$

and initial conditions $\mathbf{U}\left(t_{0}\right)=\mathbf{u}_{h}^{0}$ and $\dot{\mathbf{U}}\left(t_{0}\right)=\mathbf{u}_{h}^{1}$. 


\subsection{Fully-discrete error estimates}

In this section, we state the fully discrete error estimates. We recall that the fully-discrete formulation of problem (4) is obtained based on employing the leap-frog scheme as in Section 4. We suppose that the assumptions made in Section 3.4 hold. By employing the same arguments as the ones presented in [57] for the scalar wave equation, one can prove that

$$
\max _{n=0}^{N}\left\|\mathbf{u}\left(t_{n}\right)-\mathbf{U}\left(t_{n}\right)\right\|_{\mathbf{L}^{2}(\Omega)} \lesssim \frac{h^{s}}{p^{m-1}}+\Delta t^{2}
$$

with $s=\min (p+1, m)$ and where the hidden constant depends on $T$, on $\mathbf{u}, \dot{\mathbf{u}}, \ddot{\mathbf{u}}, \boldsymbol{\sigma}(\mathbf{u})$ and on the material properties. In particular, the $\mathbf{L}^{2}$-error estimates of Theorem 3.3 are required along with the following inequality

$$
\widetilde{\mathcal{B}}(\mathbf{v}, \mathbf{v}) \lesssim \frac{p^{4}}{h^{2}}\|\mathbf{v}\|_{\mathbf{L}^{2}(\Omega)}^{2} \quad \forall \mathbf{v} \in \mathbf{V}_{h p}
$$

that can be shown by employing inverse-trace estimates as in [28, 26] provided that the mesh satisfies suitable additional assumptions, cf. [26].

\section{Dispersion analysis}

In this section we investigate the approximation properties of the numerical scheme presented before by analyzing the dispersion errors, working in two-dimensions, i.e. $d=2$. We recall that dispersion effects arise when the numerical wave shows a phase leg with respect to the physical one. Due to the nature of the elastic wave field, in the following we will measure the dispersion errors for both $\mathrm{P}$ and $\mathrm{S}$ waves. Moreover, as a standard assumption for the plane wave analysis, see for instance [58, 59, 60], we assume that the medium is isotropic, homogeneous, unbounded and source free. We remark that, for realistic geophysical applications these assumptions are not expected to be satisfied. However, this tool provides important information to determine the discretization parameters to be used for the numerical simulation.

To study the dispersion errors of numerical schemes applied to the wave equation (1), it is convenient to consider particular solutions of the form

$$
\mathbf{u}(\mathbf{x}, t)=\mathbf{A} e^{i(\mathbf{k} \cdot \mathbf{x}-\omega t)},
$$

where $\mathbf{A}=\left[A_{1}, A_{2}\right]^{T}$ represents the amplitude of the wave, $\omega$ the angular frequency and $\mathbf{k}=$ $2 \pi / L(\cos \theta, \sin \theta)$ the wavenumber vector, $L$ being the wave length and $\theta$ the angle between the direction of propagation and the coordinate axes. The physical wave is recovered by taking the real part of 41 . Under these conditions the semi discrete problem 38 becomes

$$
M \ddot{\mathbf{U}}+B \mathbf{U}=\mathbf{0},
$$




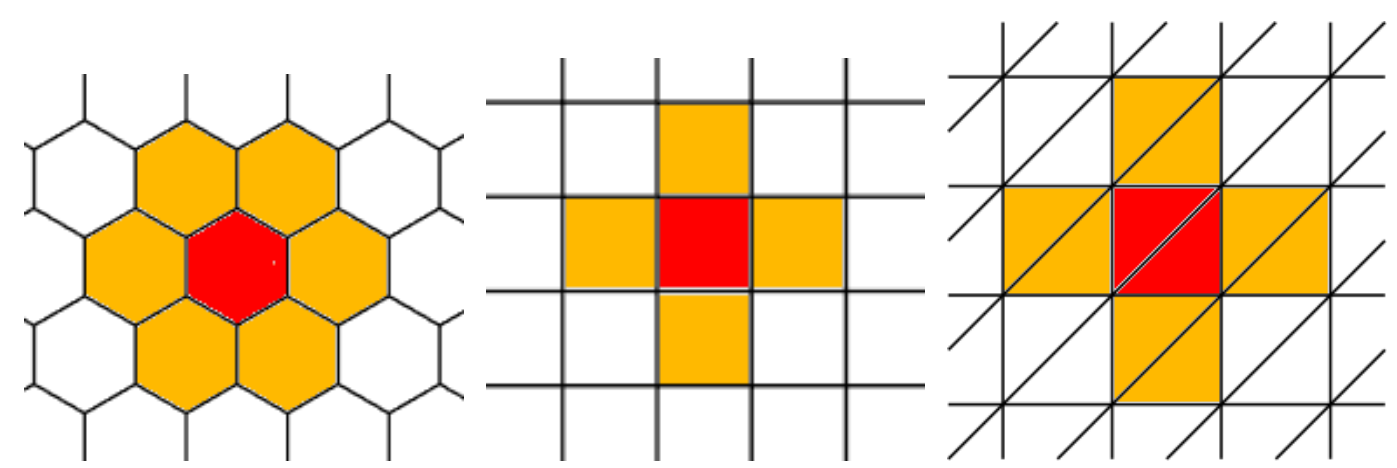

Figure 1: Periodic reference element $E_{r e f}(\mathrm{red})$ and periodic reference patterns (orange). Hexagonal (left), quadrilateral (center) and triangular grids (right).

where $\mathbf{U}\left(t_{0}\right)=\mathbf{A} e^{i(\mathbf{k} \cdot \mathbf{x})}$ and $\dot{\mathbf{U}}\left(t_{0}\right)=-i \omega \mathbf{A} e^{i(\mathbf{k} \cdot \mathbf{x})}$.

To comply with unboundedness, we consider problem (42) posed over a reference element $E_{r e f}$ (cf. Figure 1) and impose periodic boundary conditions on its boundary. Note that $E_{r e f}$ can be either a hexagon, a square or union of two triangles having uniform size $h$. Given that, the interelement jump and average contributions are assembled at the interfaces between $E_{\text {ref }}$ and its neighbors (periodic reference pattern), see Figure 1. Following the approach of [5, 8, 61, 62, 63, 11] we impose periodic boundary conditions by introducing a suitable projection matrix $P$ and we obtain from 42

$$
\widetilde{M} \ddot{\mathbf{U}}(t)+\widetilde{B} \mathbf{U}(t)=\mathbf{0}
$$

where $\widetilde{M}=P^{T} M P$ and $B=P^{T} B P$. We next consider the fully discrete formulation based on employing the leap-frog time integration scheme (39) to (43). Following [64, we substitute 44] into 43 and we obtain

$$
\widetilde{M}\left(2-e^{-i \omega \Delta t}-e^{i \omega \Delta t}\right) \frac{4}{\Delta t^{2}} \mathbf{U}\left(t_{0}\right)=\widetilde{B} \mathbf{U}\left(t_{0}\right)
$$

The above system can be rewritten as

$$
\widetilde{B} \mathbf{U}\left(t_{0}\right)=\Lambda \widetilde{M} \mathbf{U}\left(t_{0}\right),
$$

where the eigenvalues $\Lambda$ are related to the angular frequency $\omega$ at which the wave travels in the grid through the relation

$$
\Lambda=\frac{4}{\Delta t^{2}} \sin ^{2}\left(\omega \frac{\Delta t}{2}\right) .
$$

We will use this after solving the eigenvalue problem in order to derive the grid-dispersion relations as it will be shown later on. We remark that for two dimensional seismic wave propagation only two eigenvalues in (44) have a physical meaning as they are related to $\mathrm{P}$ and $\mathrm{S}$ waves, cf. 65, 5]. All the other eigenvalues correspond to nonphysical modes, see e.g. 58 for the one dimensional 
case. Therefore, the relative dispersion errors are given by

$$
e_{P}=\frac{c_{P, h}}{c_{P}}-1, \quad e_{S}=\frac{c_{S, h}}{c_{S}}-1
$$

where $c_{P, h}$ and $c_{S, h}$ are the $\mathrm{P}$ and $\mathrm{S}$ numerical wave velocities whose expression is given by

$$
c_{P, h}=\frac{h \omega_{P, h}}{2 \pi \delta r}, \quad c_{S, h}=\frac{h \omega_{S, h}}{2 \pi \delta}
$$

where $\delta=h /(p L)$ is the sampling ratio, i.e., $\delta^{-1}$ is the number of grid points per wavelength, $h$ is the mesh size, $r=c_{P} / c_{S}$ and $\omega_{P, h}$ and $\omega_{S, h}$ are the numerical angular frequencies computed through 45 for the $\mathrm{P}$ and $\mathrm{S}$ waves, respectively. First, we numerically solve (44) to obtain the eigenvalues in (45), then we compute the numerical velocities obtained for each eigenvalue and finally we compare them to the real values of $c_{P}$ and $c_{S}$, respectively.

Before analyzing the dispersion properties of the fully discrete approximation (39)-40 we want to address the stability properties of the leap-frog time integration scheme. We consider the Courant, Friedrichs and Lewy (CFL) condition

$$
\Delta t \leq C_{c f l}(p) \frac{h}{c_{P}}
$$

and we study the dependency of the constant $C_{c f l} \in(0,1)$ on the parameters involved in the model (i.e., $\lambda$ and $\mu$ ) and on the polynomial degree $p$. To this aim, by employing a scaling argument we can rewrite system (44) as

$$
\widehat{\mathcal{M}} U_{0}=\Lambda^{\prime} \widehat{\mathcal{M}} U_{0}
$$

where $\Lambda^{\prime}=(h / \Delta t)^{2} \sin ^{2}\left(\omega_{h} \Delta t / 2\right)$. Following, e.g., 61] we define the stability parameter $q$, as

$$
q=c_{P} \frac{\Delta t}{h}
$$

and we derive the stability bound

$$
q \leq \frac{c_{P}}{\sqrt{\Lambda^{\prime}}}=C_{c f l}\left(\Lambda^{\prime}\right)
$$

As stated in [66], the eigenvalue $\Lambda^{\prime}$ depends on the wavenumber vector $\mathbf{k}$ and therefore on the value of the angle $\theta$. Thus, condition 50 can be reformulated as

$$
q \leq c^{*}(\lambda, \mu, \eta) \frac{1}{\sqrt{\Lambda_{\max }^{\prime}}}=q_{c f l},
$$

where $\Lambda_{\text {max }}^{\prime}$ is the maximum eigenvalue of $(49)$, taken with respect to the values of $\theta$. The constant 


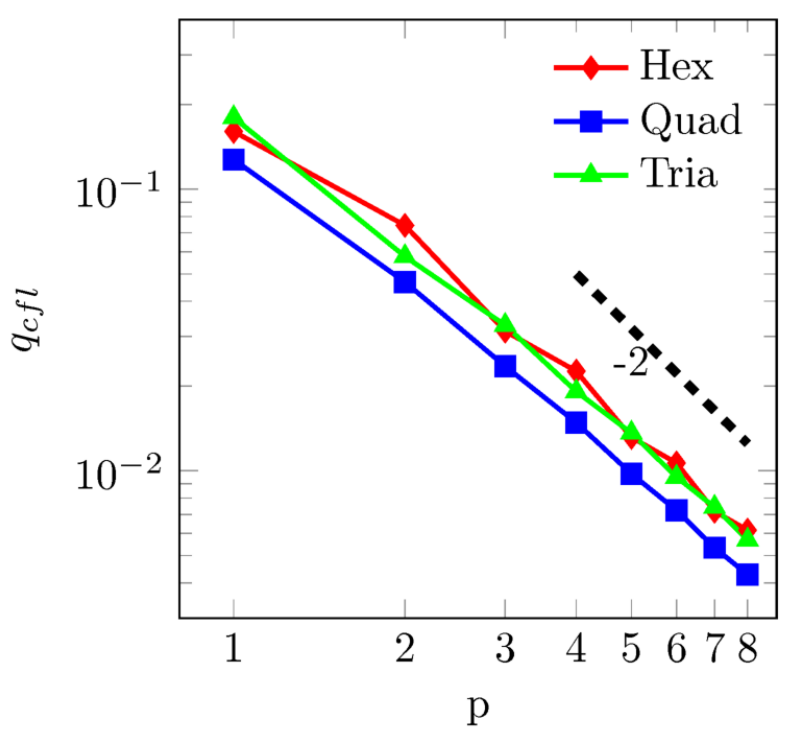

Figure 2: Stability parameter $q_{c f l}$ versus the polynomial degree $p$ on triangular, quadrilateral and hexagonal decomposition. The black dotted line corresponds to the asymptotic trend $p^{-2}$.

$c^{*}$ depends on the Lamé parameters $\lambda$ and $\mu$ and it is proportional to $\eta^{-1 / 2}$, see 67 .

\subsection{Numerical dispersion analysis}

We first give a quantitative estimate of the parameter $q_{c f l}$ appearing in (51), supposing that a uniform polynomial approximation degree is employed on all mesh elements, i.e., $p_{\kappa}=p$, for any $\kappa \in \mathcal{T}$. We set $c_{P}=1, \delta=0.2$ and $r=2$. Similar results can be obtained for different values of $r$, see [1].

In Figure 2 we observe that, for all grids, the value of $q_{c f l}$ decays proportionally to $p^{-2}$, in agreement with 66, 8, 63, 61, 11. In addition, we notice that, for a given polynomial degree, quadrilateral elements are subjected to a more restrictive stability condition, i.e., lower values of $q_{c f l}$ are obtained. In particular, in the case of a discretization based on a triangular grid (resp. hexagonal grid), the stability parameter $q_{c f l}$ is 1.3 larger (resp. 1.4) of the corresponding value computed on a quadrilateral mesh.

Now, we present the dispersion analysis for the fully discrete approximation, varying the discretization parameters $p, \delta$ and $q$. We first address the behavior of the dispersion error with respect to the sampling ratio $\delta$, fixing $p=4$ and $\theta=\pi / 4$. We consider the relative stability parameter $q_{r e l}=q / q_{c l f}$ in the range $[0.1,1]$. Notice that the value $q_{c f l}$ has been computed in agreement with 51]. As expected, when $q_{r e l}$ approaches zero, i.e., $\Delta t$ goes to 0 , the fully discrete curves recover the semi discrete ones (see Figure 3). In Figure 4 we compare the results 
obtained with all the different tasselations for $q_{r e l}=0.1$. We observe that the numerical schemes retain the same level of accuracy. In particular, for $\delta<0.2$, i.e., with more than five points per wavelength, all discretizations produce negligible dispersion errors, i.e., less than $10^{-6}$. Next, we analyze the dispersion error by varying the polynomial degree $p$, fixing $\delta=0.2$ and $\theta=\pi / 4$. In Figure 5 we retrieve the exponential convergence observed in the semi-discrete case (red line) as $q_{\text {rel }}$ goes to zero. Indeed, for sufficiently small values of $q$, the following asymptotic relation holds $\omega_{h} \approx \sqrt{\Lambda}+\mathcal{O}\left(\Delta t^{2}\right)$, see [63]. Therefore $\omega_{h}$ decays as in the semi discrete case until the term $\Delta t^{2}$ becomes dominant. In Figure 6 we compare the behavior of the fully discrete scheme for the error smaller than $10^{-6}$ ) is obtained for $p \geq 5$.

Finally, we study the dispersion errors as a function of the angle $\theta$ in the parameter $\mathbf{k}$ of (41). In Figure 7 we report the results obtained for $p=4, \delta=0.2$ and $q_{r e l}=0.1$. We notice that with hexagonal and quadrilateral grids the error behaves symmetrically with respect to the origin of the axes, whereas with triangular grids the error grows along the direction given by the diagonal, cf. [11, 61.

\section{Numerical results}

In this section we verify the convergence estimates proved in Section 3 and we present a geophysical application of elastic wave propagation in a heterogeneous (anisotropic) medium.

\subsection{Benchmark test case}

We first solve the wave propagation problem in $\Omega=(0,1)^{2}$, choosing $\lambda=\mu=\rho=1$ and assuming that the exact solution of (1) is given by

$$
\mathbf{u}(\mathbf{x}, t)=\sin (\sqrt{2} \pi t)\left[\begin{array}{c}
-\sin (\pi x)^{2} \sin (2 \pi y) \\
\sin (2 \pi x) \sin (\pi y)^{2}
\end{array}\right] .
$$

Dirichlet boundary conditions and initial conditions are set accordingly. For the analysis we fix the final time $T=1$ and a time step $\Delta t=10^{-4}$. We compute the error $\left\|\mathbf{u}-\mathbf{u}_{h}\right\|_{\mathrm{E}}$ by varying the polynomial degree $p_{\kappa}=p$, for any $\kappa \in \mathcal{T}$, and the number of polygonal elements $N_{e l}$. We consider two mesh configurations and four level of refinement for each grid as shown in Figure 8 In Figure 9 (left), resp. Figure 10 (left), we show the computed error $\left\|\mathbf{u}(T)-\mathbf{u}_{h}(T)\right\|_{\mathrm{E}}$ obtained on a shape-regular grid (cf. Figure 8 a)), resp. distorted grid (cf. Figure 8 b)), versus the polynomial degree $p$, which varies from 1 to 7 , in semilogarithmic scale. The number of polygonal elements for these grids is fixed to 160 . We observe the exponential converge in $p$, since the chosen solution is analytic. The computed error is also tested versus the number of degrees of freedom, as shown 

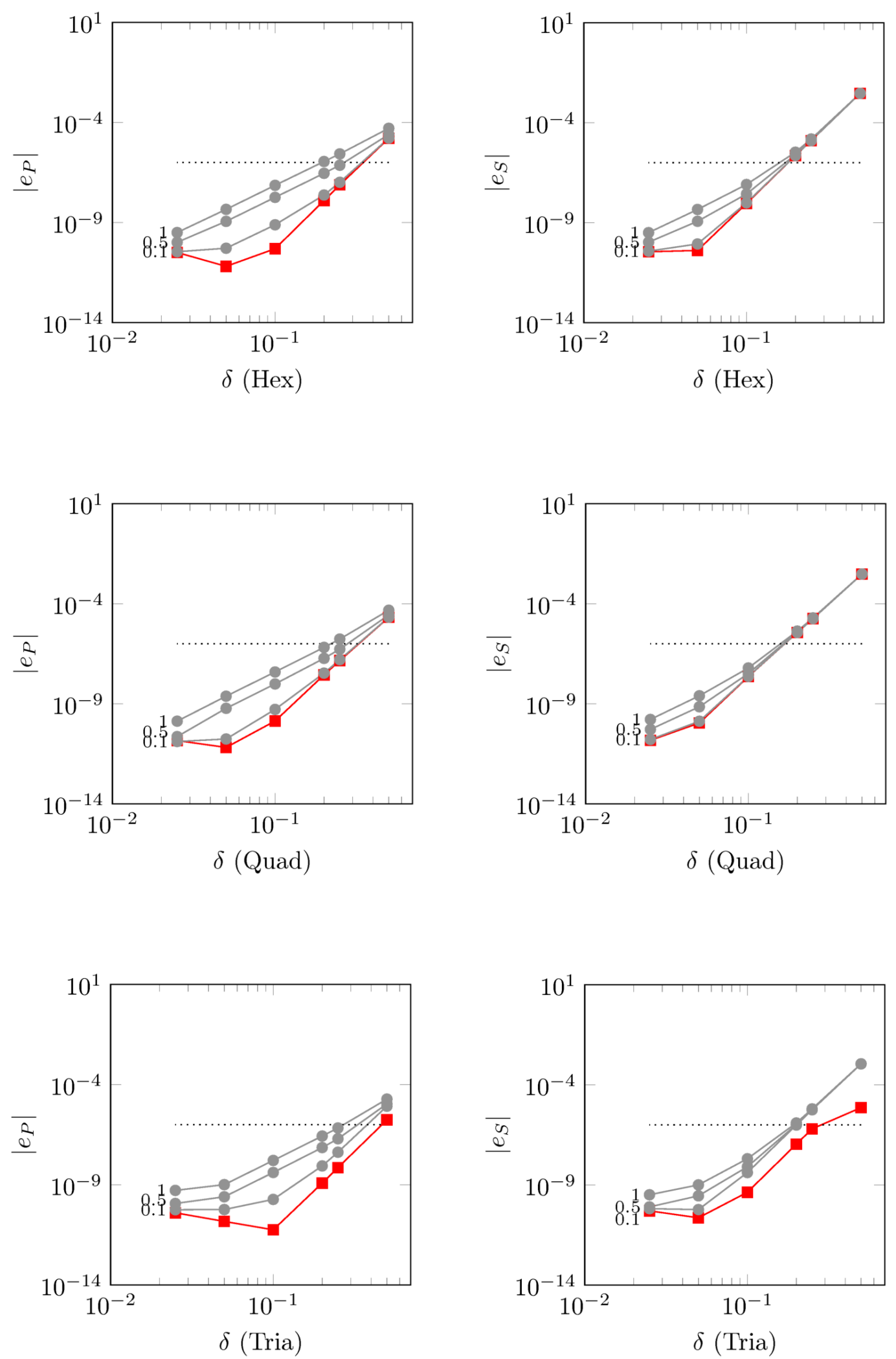

Figure 3: Dispersion errors $\left|e_{P}\right|$ (left) and $\left|e_{S}\right|$ (right) as a function of $\delta$ for $p=4$. The square marked lines are obtained with analytical time integration. The circle marked lines refer to the fully discrete approximation with $q_{\text {rel }}=0.1,0.5,1$. 

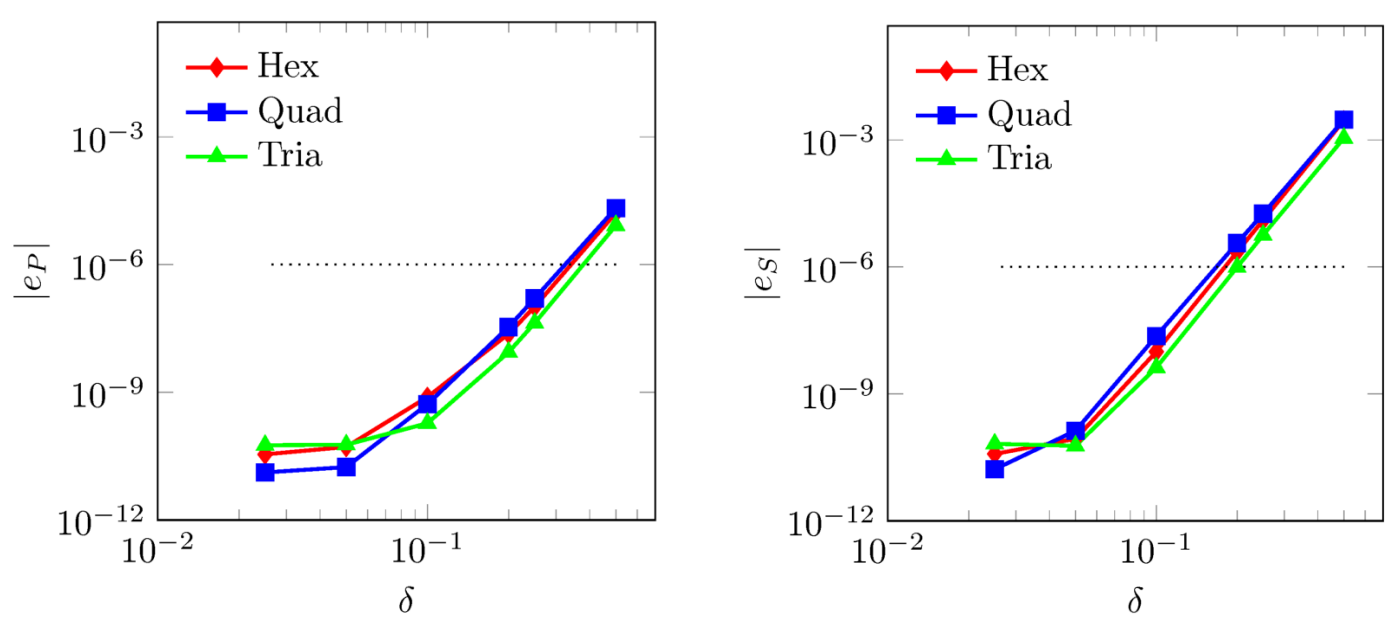

Figure 4: Dispersion errors $\left|e_{P}\right|$ (left) and $\left|e_{S}\right|$ (right) as a function of $\delta$, fixing $p=4$ and $q_{r e l}=0.1$.

Table 1: Coefficients for the heterogeneous anisotropic model given in $\left[10^{7} \mathrm{~N} / \mathrm{m}^{2}\right]$ for the anisotropic and isotropic materials. The material density $\rho$ is given in $\left[\mathrm{kg} / \mathrm{m}^{3}\right]$

\begin{tabular}{lccccc}
\hline & $\rho$ & $\mathcal{D}_{11}$ & $\mathcal{D}_{12}$ & $\mathcal{D}_{22}$ & $\mathcal{D}_{33}$ \\
\hline Isotropic & 2000 & 5.9858 & 1.9858 & 5.9858 & 2 \\
Anisotropic & 2000 & 5.9858 & 0.6017 & 2.2492 & 2 \\
\hline
\end{tabular}

in Figure 9 (right), resp. Figure 10 (right). Here, we notice that for $p=2,3,4$ we retrieve the algebraic convergence proved in 29 for both the grid configurations shown in Figure 8.

\subsection{Elastic wave propagation in an anisotropic medium}

As an application of the presented method, we study the elastic wave propagation in a heterogeneous medium. The computational domain $\Omega=(-500,500) m \times(-500,500) m$ contains two materials separated by a straight line at $y=0$. In the upper part $(y>0)$ we have an anisotropic (transversely isotropic) body with the symmetry axis in the $x$-direction, whereas in the lower part $(y<0)$ we use an isotropic material. Analogous test cases regarding wave propagation in anisotropic media can be found for instance in [10, 68, 69, 70, In this case, the stiffness tensor $\mathcal{D}$ has 4 independent components. Using the reduced Voigt notation (see e.g., [71]), Hooke's law (2) becomes

$$
\left(\begin{array}{c}
\sigma_{11} \\
\sigma_{22} \\
\sigma_{12}
\end{array}\right)=\left(\begin{array}{ccc}
\mathcal{D}_{11} & \mathcal{D}_{12} & 0 \\
\mathcal{D}_{12} & \mathcal{D}_{22} & 0 \\
0 & 0 & \mathcal{D}_{33}
\end{array}\right)\left(\begin{array}{c}
\epsilon_{11} \\
\epsilon_{22} \\
2 \epsilon_{12}
\end{array}\right)
$$

Then, the isotropic case can be easily obtained by letting $\mathcal{D}_{11}=\mathcal{D}_{22}=\lambda+2 \mu, \mathcal{D}_{12}=\lambda$ and $\mathcal{D}_{33}=\mu$. In Table 1 we report $\mathrm{i}$ the mechanical properties of the materials. The source is 

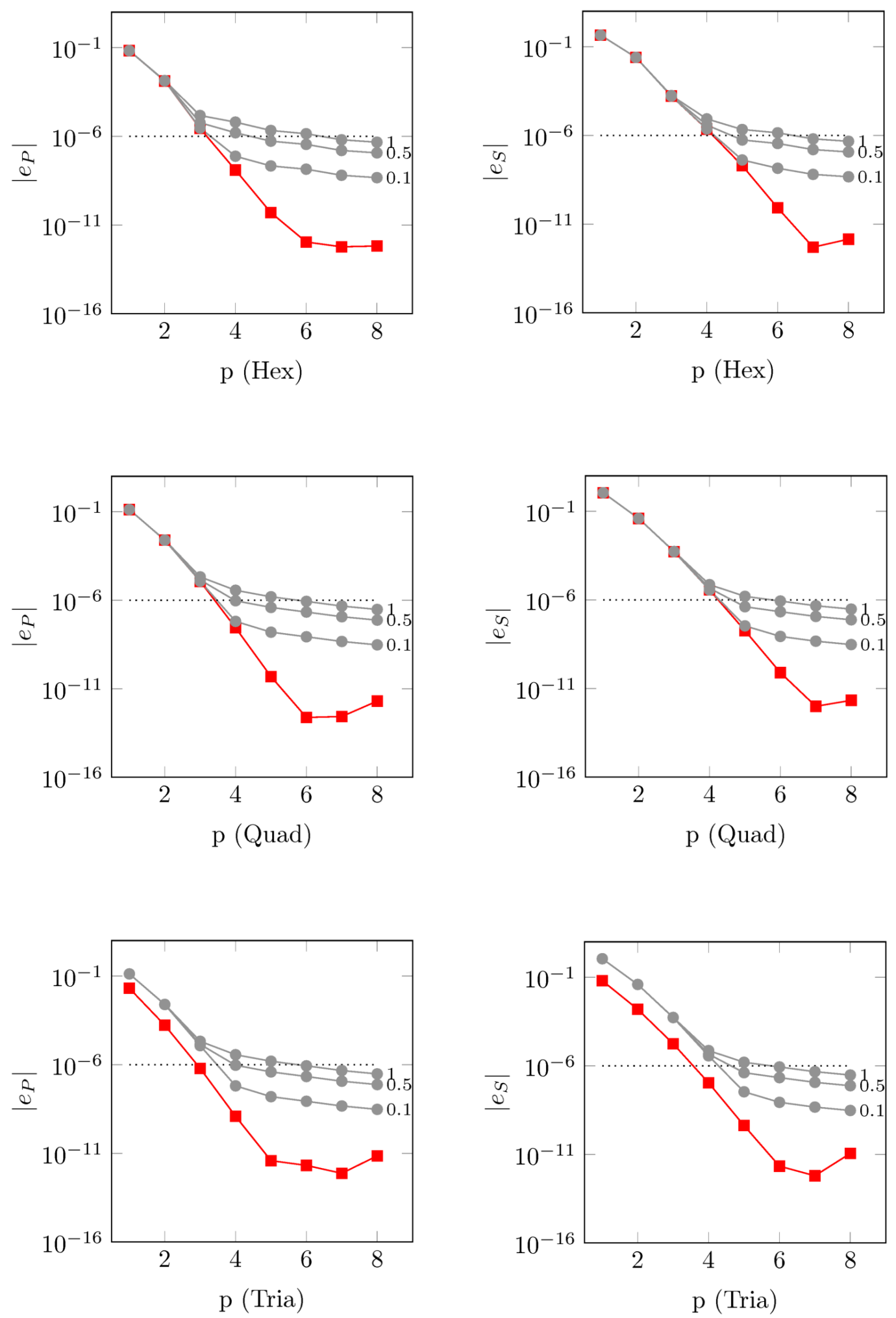

Figure 5: Dispersion errors $\left|e_{P}\right|$ (left) and $\left|e_{S}\right|$ (right) as a function of $p$ for $p=4$. The square marked lines are obtained with analytical time integration. The circle marked lines refer to the fully discrete approximation with $q_{\text {rel }}=0.1,0.5,1$. 

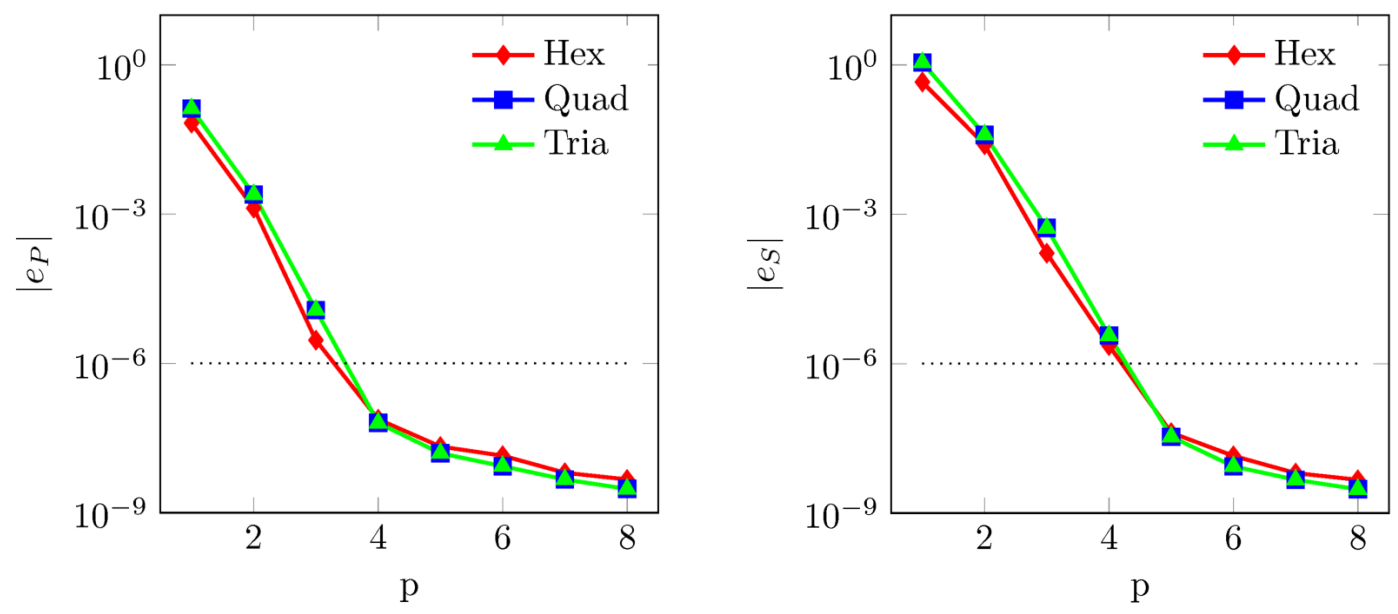

Figure 6: Dispersion errors $\left|e_{P}\right|$ (left) and $\left|e_{S}\right|$ (right) as a function of $p$ with $q_{\text {rel }}=0.1$.

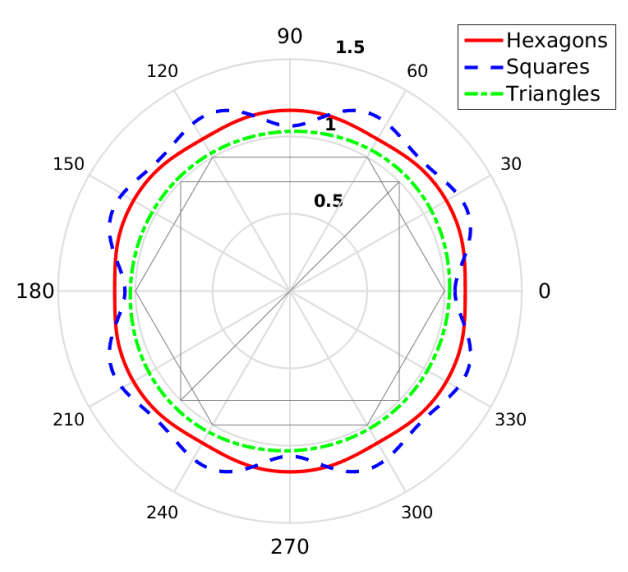

(a) $e_{P}$

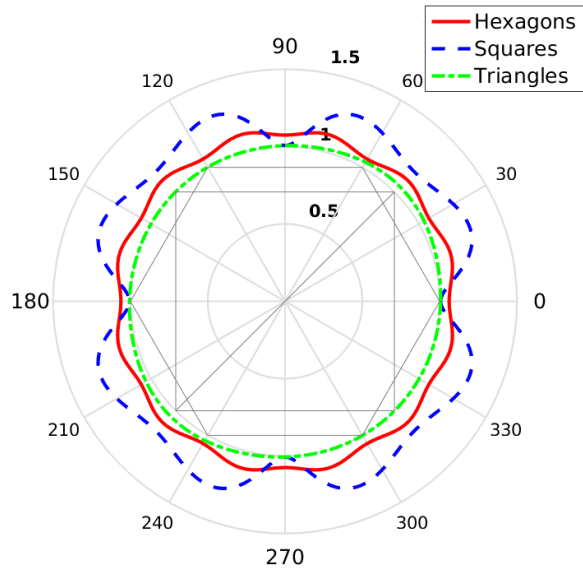

(b) $e_{S}$

Figure 7: Dispersion errors $e_{P}$ (left) and $e_{S}$ (right) as a function of the incidence angle $\theta$. For visualization purposes the results have been magnified by a factor $5 \cdot 10^{7}$ and $2 \cdot 10^{5}$, respectively.

a)

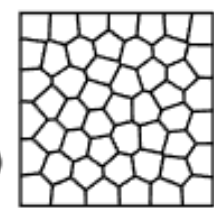

b)

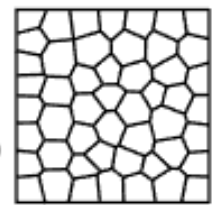

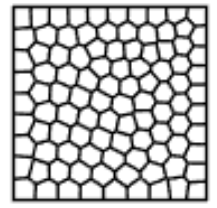

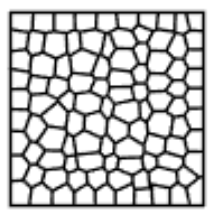

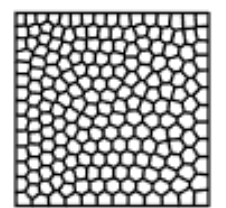
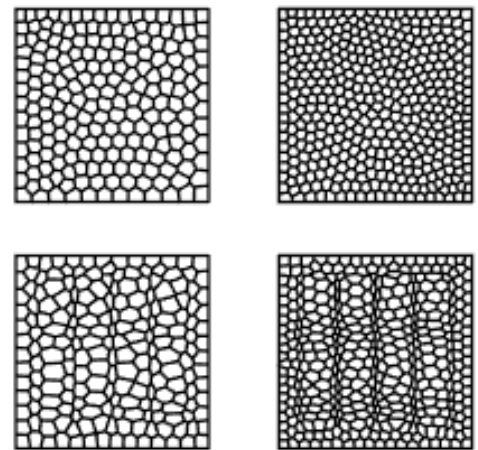

Figure 8: Different mesh configurations considered with increasing number of polygonal elements from 50 (left) to 400 (right). Shape-regular (a) vs. distorted (b) grids. 

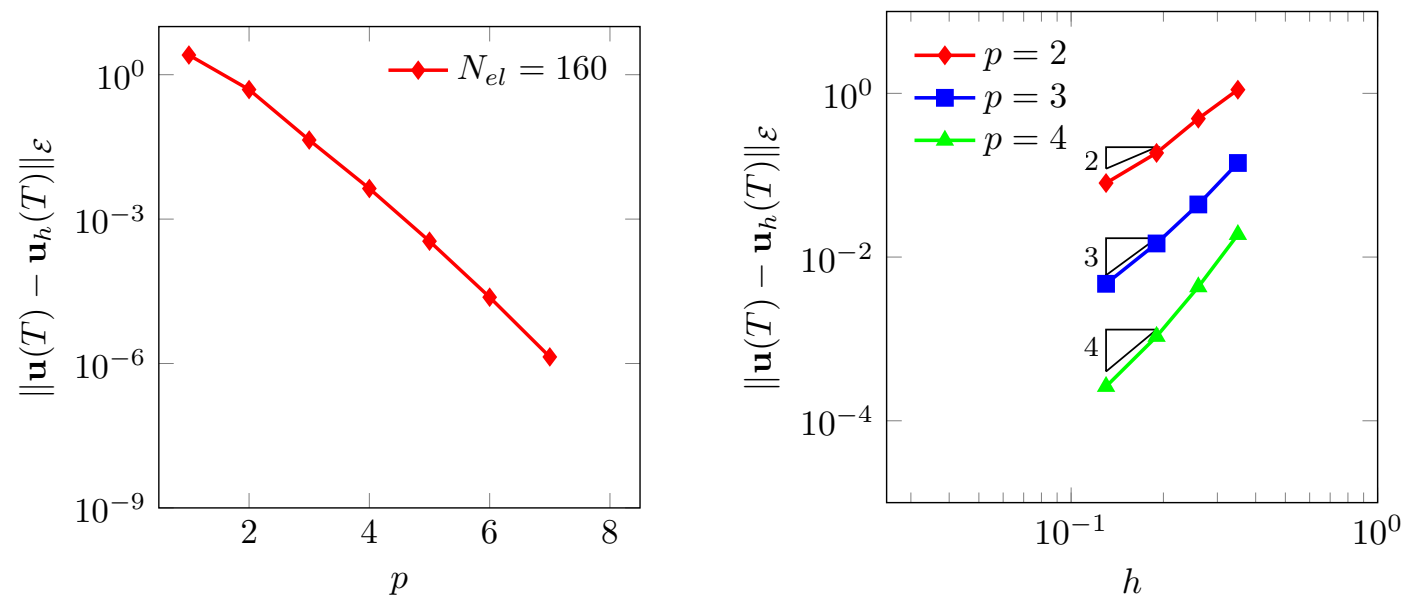

Figure 9: Grid a): computed error $\left\|\mathbf{u}(T)-\mathbf{u}_{h}(T)\right\|_{\mathrm{E}}$ versus the polynomial degree $p$, fixing $N_{e l}=160$ (left) and versus the mesh size $h=1 / N_{e l}, N_{e l}=50,100,200,400$ (right) fixing $p=2,3,4$. Results are obtained choosing as observation time $T=1$, with $\Delta t=10^{-4}$.
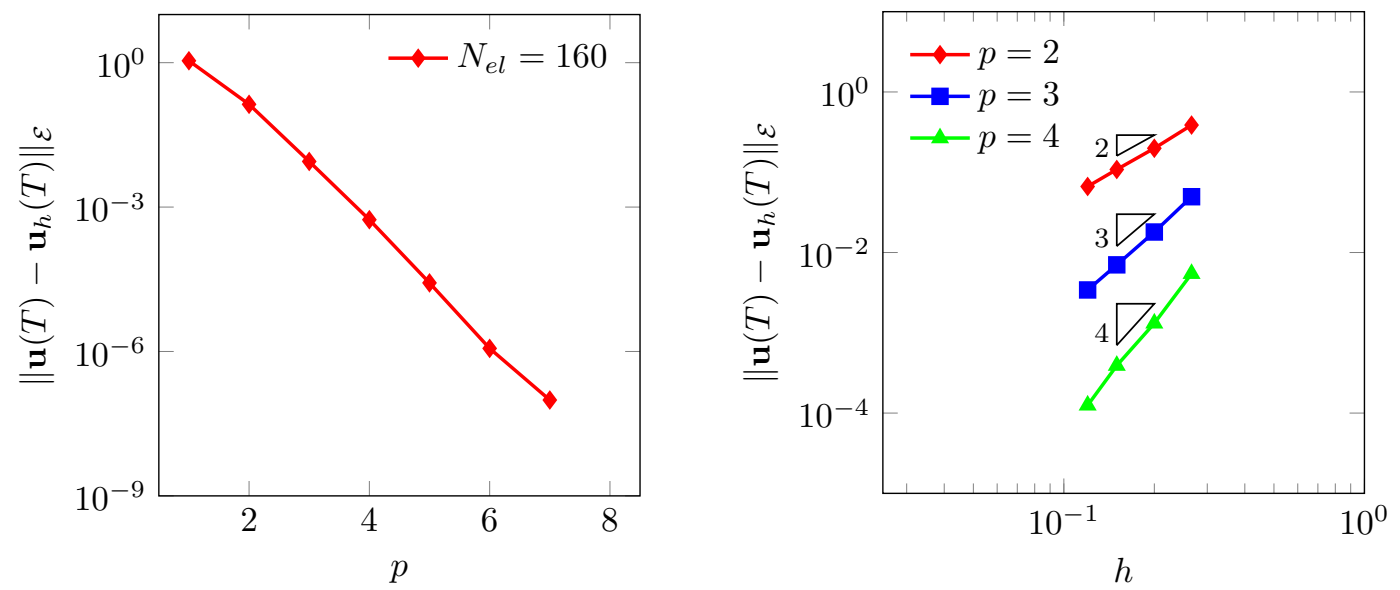

Figure 10: Grid b): computed error $\left\|\mathbf{u}(T)-\mathbf{u}_{h}(T)\right\|_{\mathrm{E}}$ versus the polynomial degree $p$, fixing $N_{e l}=160$ (left) and versus the mesh size $h=1 / N_{e l}, N_{e l}=50,100,200,400$ (right) fixing $p=2,3,4$. Results are obtained choosing as observation time $T=1$, with $\Delta t=10^{-4}$. 

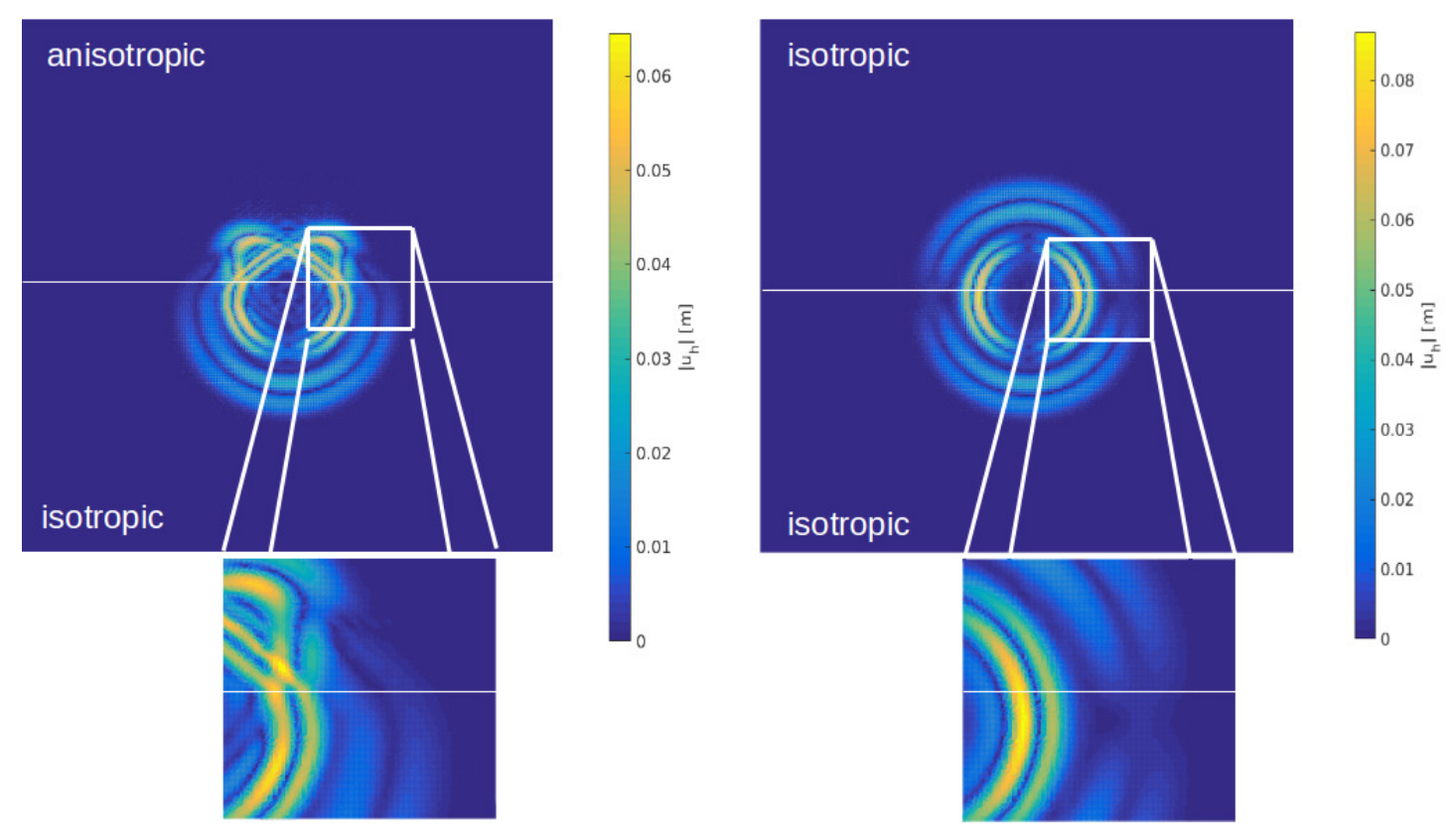

Figure 11: Displacement field $\left|\mathbf{u}_{h}\right|$ at time $t=2 s$ : heterogeneous materials (left) and homogeneous material (right).

represented by a vertical force of the form

$$
\mathbf{f}(\mathbf{x}, t)=\left(0, \phi(t) e^{-\left\|\mathbf{x}-\mathbf{x}_{s}\right\|^{2}}\right)^{T}
$$

where $\mathbf{x}_{s}=(0,-25) \mathrm{m}$, that is $25 \mathrm{~m}$ below the material interface inside the isotropic material and is acting in the y-direction and $\phi(t)=10^{7}\left(1-8 \pi^{2}(t-1)^{2}\right) e^{-4 \pi(t-1)^{2}}$. On the boundaries of the domain we impose a null displacement, i.e, $\mathbf{u}=\mathbf{0}$. For the spatial discretization we employ fifth order polynomials, i.e. $p_{\kappa}=5$ for any $\kappa \in \mathcal{T}$ on a polygonal grid with size of approximately $30 \mathrm{~m}$. The time integration is carried out by using the leap-frog scheme (39)- 40 and fixing the time step $\Delta t=5 \cdot 10^{-4} \mathrm{~s}$ for a total observation time $T=5.5 \mathrm{~s}$. For a qualitative comparison we report in Figure 11 the modulus of the approximated solution $\left|\mathbf{u}_{h}\right|$ obtained in the case of a heterogeneous anisotropic (left) and pure a isotropic (right) medium, respectively. Finally, the computed horizontal and vertical displacements at the receiver location $R_{1}=(150,125) \mathrm{m}$ are plotted in Figure 12 for the isotropic (top) and anisotropic (bottom) case. From the plot we can clearly distinguish the different arrival of the P- and S-waves. Indeed in the anisotropic medium the former is delayed of about $0.5 s$ while the latter of about $2 s$. Moreover, due to the presence of anisotropy the amplitude of the wave field is reduced. 


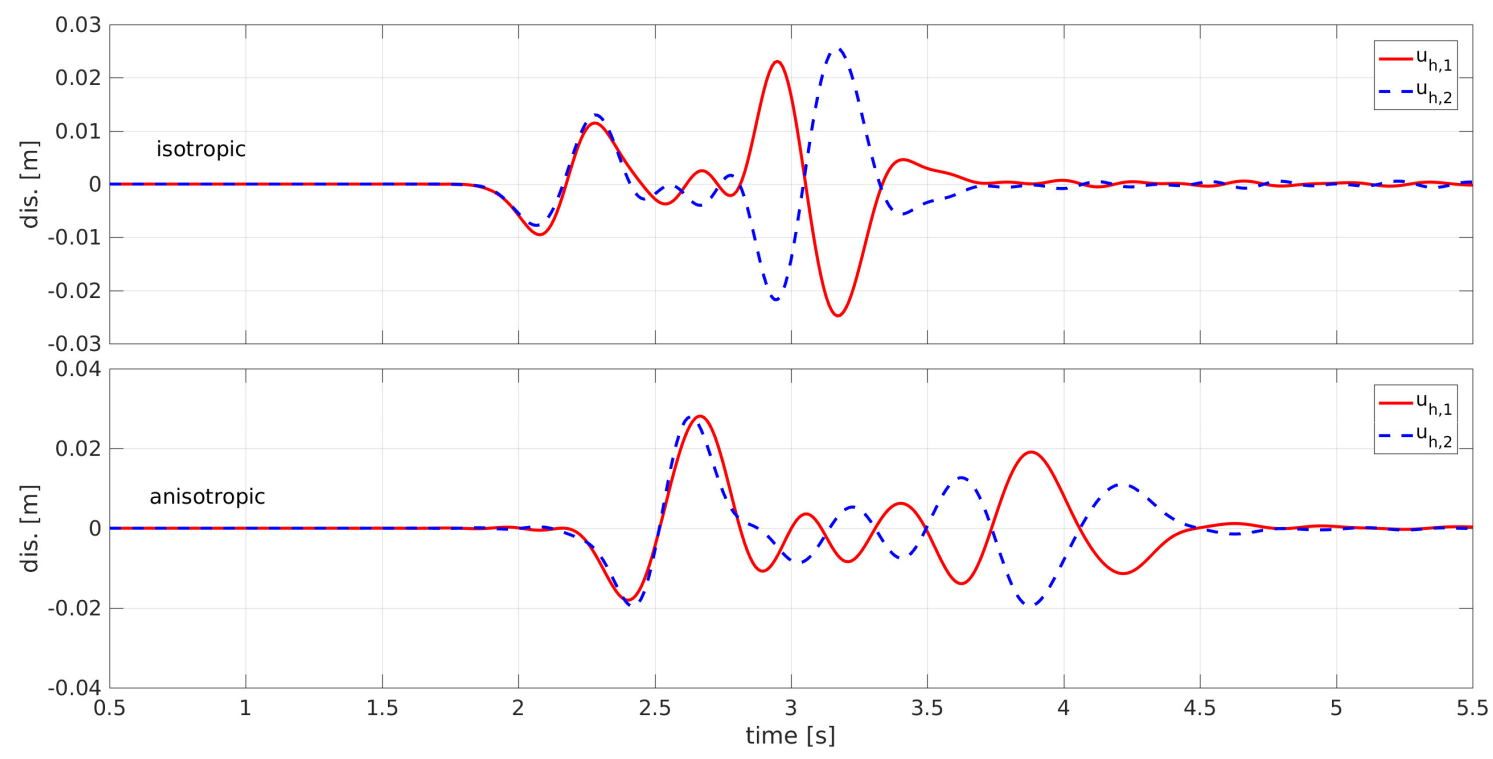

Figure 12: Computed horizontal and vertical displacements at the receiver location $R_{1}=(150,125) \mathrm{m}$ : isotropic (top) and anisotropic (bottom) case.

\section{Conclusions}

We proposed a high-order Discontinuous Galerkin method for the approximate solution of the elastodynamics equations on computational meshes made by general polygonal/polyhedral elements. We analyzed the well-posedness of the numerical formulation and proved $h p$-version error estimates for the semi-discrete and the fully discrete numerical scheme, the latter being obtained based on employing the leap-frog scheme for time discretization. In particular, error bounds in a mesh-dependent energy norm and the $L^{2}$-norm were derived for the semi-discrete and fully discrete formulation, respectively. A thorough comparison with standard Discontinuous Galerkin schemes on simpicial/quadrilateral grids is presented in term of a dispersion and stability analysis. We showed that polygonal meshes behave as classical simplicial/quadrilateral grids in terms of dispersion and stability properties. Finally, we applied the scheme to different twodimensional test cases. Here, the flexibility in the process of mesh design offered by polytopic elements can be fully exploited.

\section{Acknowledgments}

The authors are grateful to the Referees for their thorough and constructive comments that have greatly contributed to the improvement of the manuscript.

\section{References}

[1] D. Komatitsch, J. Tromp, Spectral-element simulations of global seismic wave propagation-I. Validation, Geophys. J. Int. 149 (2) (2002) 390-412. 
[2] D. Komatitsch, J. Tromp, Spectral-element simulations of global seismic wave propagationII. Three-dimensional models, oceans, rotation and self-gravitation, Geophys. J. Int. 150 (1) (2002) 303-318.

[3] B. Rivière, M. F. Wheeler, Discontinuous finite element methods for acoustic and elastic wave problems, Contemporary Mathematics 329 (2003) 271-282.

[4] B. Rivière, S. Shaw, M. F. Wheeler, J. R. Whiteman, Discontinuous Galerkin finite element methods for linear elasticity and quasistatic linear viscoelasticity, Numer. Math. 95 (2) (2003) $347-376$.

[5] J. D. De Basabe, M. K. Sen, M. F. Wheeler, The interior penalty discontinuous Galerkin method for elastic wave propagation: grid dispersion, Geophys. J. Int. 175 (1) (2008) 83-93.

[6] M. Käser, M. Dumbser, An arbitrary high-order discontinuous Galerkin method for elastic waves on unstructured meshes - I: The two-dimensional isotropic case with external source terms, Geophys. J. Int. 166 (2) (2006) 855-877.

[7] M. Dumbser, M. Käser, An arbitrary high-order discontinuous Galerkin method for elastic waves on unstructured meshes - II. The three-dimensional isotropic case, Geophys. J. Int. 167 (1) (2006) 319-336.

[8] P. F. Antonietti, I. Mazzieri, A. Quarteroni, F. Rapetti, Non-conforming high order approximations of the elastodynamics equation, Comput. Methods Appl. Mech. Engrg. 209 (2012) 212-238.

[9] E. D. Mercerat, N. Glinsky, A nodal high-order discontinuous Galerkin method for elastic wave propagation in arbitrary heterogeneous media, Geophys. J. Int. 201 (2) (2015) 11011118.

[10] P. F. Antonietti, B. Ayuso de Dios, I. Mazzieri, A. Quarteroni, Stability analysis of discontinuous Galerkin approximations to the elastodynamics problem, J. Sci. Comput. 68 (2016) $143-170$.

[11] A. Ferroni, P. F. Antonietti, I. Mazzieri, A. Quarteroni, Dispersion-dissipation analysis of 3D continuous and discontinuous spectral element methods for the elastodynamics equation, Geophys. J. Int. 211 (3) (2017) 1554-1574.

[12] P. F. Antonietti, A. Ferroni, I. Mazzieri, R. Paolucci, A. Quarteroni, C. Smerzini, M. Stupazzini, Numerical modeling of seismic waves by Discontinuous Spectral Element methods, To appear, ESAIM Proceedings and Surveys (2017). 
[13] P. F. Antonietti, A. Ferroni, I. Mazzieri, A. Quarteroni, hp-version Discontinuous Galerkin approximations of the elastodynamics equation, Lecture Notes in Computational Science and Engineering 119 (2017) 3-19.

[14] B. Rivière, Discontinuous Galerkin methods for solving elliptic and parabolic equations: theory and implementation, Society for Industrial and Applied Mathematics, 2008.

[15] J. S. Hesthaven, T. Warburton, Nodal discontinuous Galerkin methods: algorithms, analysis, and applications, Springer Science \& Business Media, 2007.

[16] D. A. Di Pietro, A. Ern, Mathematical aspects of discontinuous Galerkin methods, Vol. 69, Springer Science \& Business Media, 2011.

[17] P. F. Antonietti, F. Brezzi, L. D. Marini, Bubble stabilization of discontinuous Galerkin methods, Comput. Methods Appl. Mech. Engrg. 198 (21-26) (2009) 1651-1659.

[18] P. F. Antonietti, S. Giani, P. Houston, hp-version composite discontinuous Galerkin methods for elliptic problems on complicated domains, SIAM J. Sci. Comput. 35 (3) (2013) A1417A1439.

[19] P. F. Antonietti, S. Giani, P. Houston, Domain decomposition preconditioners for Discontinuous Galerkin methods for elliptic problems on complicated domains, J. Sci. Comput. 60 (1) (2014) 203-227.

[20] A. Cangiani, E. H. Georgoulis, P. Houston, $h p$-Version discontinuous Galerkin methods on polygonal and polyhedral meshes, Math. Models Methods Appl. Sci. 24 (10) (2014) $2009-$ 2041.

[21] F. Bassi, L. Botti, A. Colombo, F. Brezzi, G. Manzini, Agglomeration-based physical frame dg discretizations: An attempt to be mesh free, Math. Mod. Meth. Appl. Sci. 24 (8) (2014) 1495-1539.

[22] A. Cangiani, Z. Dong, E. H. Georgoulis, $h p$-version space-time discontinuous Galerkin methods for parabolic problems on prismatic meshes, SIAM J. Sci. Comput. 39 (4) (2017) A1251A1279.

[23] A. Cangiani, Z. Dong, E. H. Georgoulis, P. Houston, $h p$-Version Discontinuous Galerkin methods for advection-diffusion-reaction problems on polytopic meshes, ESAIM Math. Model. Numer. Anal. 50 (2016) $699-725$.

[24] P. F. Antonietti, A. Cangiani, J. Collis, Z. Dong, E. Georgoulis, S. Giani, P. Houston, Review of discontinuous Galerkin finite element methods for partial differential equations on 
complicated domains, Lecture Notes in Computational Science and Engineering 114 (2016) $279-308$.

[25] P. F. Antonietti, C. Facciolà, A. Russo, M. Verani, Discontinuous Galerkin approximation of flows in fractured porous media, MOX Report 22/2016, Submitted.

[26] P. F. Antonietti, P. Houston, X. Hu, M. Sarti, M. Verani, Multigrid algorithms for hp-version interior penalty discontinuous galerkin methods on polygonal and polyhedral meshes, Calcolo (2017) $1-30$.

[27] P. F. Antonietti, G. Pennesi, V-cycle multigrid algorithms for discontinuous Galerkin methods on non-nested polytopic meshes, MOX report 49/2017, submitted.

[28] A. Cangiani, Z. Dong, E. H. Georgoulis, P. Houston, hp-Version Discontinuous Galerkin Methods on Polygonal and Polyhedral Meshes, SpringerBriefs in Mathematics, 2017.

[29] L. Beirão da Veiga, F. Brezzi, A. Cangiani, G. Manzini, L. D. Marini, A. Russo, Basic principles of virtual element methods, Math. Models Methods Appl. Sci. 23 (1) (2013) 199 214.

[30] L. Beirão da Veiga, F. Brezzi, L. D. Marini, A. Russo, Mixed virtual element methods for general second order elliptic problems on polygonal meshes, ESAIM Math. Model. Numer. Anal. 50 (3) (2016) $727-747$.

[31] L. Beirão da Veiga, F. Brezzi, L. D. Marini, A. Russo, Virtual element method for general second-order elliptic problems on polygonal meshes, Math. Models Methods Appl. Sci. 26 (4) (2016) $729-750$.

[32] P. F. Antonietti, L. Beirão da Veiga, D. Mora, M. Verani, A stream virtual element formulation of the Stokes problem on polygonal meshes, SIAM J. Numer. Anal. 52 (1) (2014) $386-$ 404.

[33] P. F. Antonietti, L. Beirão da Veiga, S. Scacchi, M. Verani, A C1 virtual element method for the Cahn-Hilliard equation with polygonal meshes, SIAM J. Numer. Anal. 54 (1) (2016) 34 $-56$.

[34] P. F. Antonietti, G. Manzini, M. Verani, The fully nonconforming Virtual Element method for biharmonic problems, M3AS Math. Models Methods Appl. Sci., to appear (2017).

[35] J. Hyman, M. Shashkov, S. Steinberg, The numerical solution of diffusion problems in strongly heterogeneous non-isotropic materials, J. Comput. Phys. 132 (1) (1997) 130 - 148. 
[36] F. Brezzi, K. Lipnikov, V. Simoncini, A family of mimetic finite difference methods on polygonal and polyhedral meshes, Math. Mod. Meth. Appl. S. 15 (10) (2005) 1533 - 1551.

[37] F. Brezzi, K. Lipnikov, M. Shashkov, Convergence of the mimetic finite difference method for diffusion problems on polyhedral meshes., SIAM J. Numer. Anal. 43 (5) (2005) 1872 - 1896 (electronic).

[38] F. Brezzi, K. Lipnikov, M. Shashkov, Convergence of mimetic finite difference method for diffusion problems on polyhedral meshes with curved faces, Math. Models Methods Appl. Sci. 16 (2) (2006) $275-297$.

[39] L. Beirão da Veiga, K. Lipnikov, G. Manzini, The mimetic finite difference method for elliptic problems, Vol. 11, Springer, Cham, 2014.

[40] P. F. Antonietti, L. Formaggia, A. Scotti, M. Verani, V. Nicola, Mimetic finite difference approximation of flows in fractured porous media, ESAIM Math. Model. Numer. Anal. 50 (3) (2016) $809-832$.

[41] W. Hackbusch, S. A. Sauter, Composite finite elements for the approximation of PDEs on domains with complicated micro-structures, Numer. Math. 75 (4) (1997) 447-472.

[42] W. Hackbusch, S. A. Sauter, Composite finite elements for problems containing small geometric details. Part II: Implementation and numerical results, Comput. Visual Sci. 1 (4) (1997) $15-25$.

[43] A. Tabarraei, N. Sukumar, Extended finite element method on polygonal and quadtree meshes, Comput. Methods Appl. Mech. Eng. 197 (5) (2008) 425-438.

[44] T.-P. Fries, T. Belytschko, The extended/generalized finite element method: An overview of the method and its applications, Internat. J. Numer. Methods Engrg. 84 (3) (2010) 253-304.

[45] N. Sukumar, A. Tabarraei, Conforming polygonal finite elements, Internat. J. Numer. Methods Engrg. 61 (12) (2004) 2045-2066.

[46] D. A. Di Pietro, A. Ern, S. Lemaire, An arbitrary-order and compact-stencil discretization of diffusion on general meshes based on local reconstruction operators, Comput. Method Appl. Math. 14 (4) (2014) $461-472$.

[47] D. A. Di Pietro, A. Ern, A hybrid high-order locking-free method for linear elasticity on general meshes, Comput. Methods Appl. Mech. Engrg. 283 (1) (2015) 1 - 21.

[48] D. A. Di Pietro, A. Ern, Hybrid high-order methods for variable-diffusion problems on general meshes, C. R. Math. Acad. Sci. Soc. R. Can. 353 (1) (2015) 31 - 34. 
[49] F. Bonaldi, D. A. Di Pietro, G. Geymonat, F. Krasucki, A hybrid high-order method for Kirchhoff-Love plate bending problems, accepted for publication in Math. Model. Numer. Anal. (2018).

[50] L. C. Evans, Partial differential equations, Vol. 19 of Graduate Studies in Mathematics, American Mathematical Society, Providence, RI, 1998.

[51] P.-A. Raviart, J.-M. Thomas, Introduction à l' analyse numérique des équations aux dérivées partielles, Masson, 1983.

[52] D. N. Arnold, F. Brezzi, R. S. Falk, L. D. Marini, Locking-free Reissner-Mindlin elements without reduced integration, Comput. Methods Appl. Mech. Engrg. 196 (37-40) (2007) 36603671.

[53] D. N. Arnold, F. Brezzi, B. Cockburn, L. D. Marini, Unified analysis of discontinuous Galerkin methods for elliptic problems, SIAM J. Numer. Anal. 39 (5) (2001/02) 1749-1779.

[54] A. Quarteroni, Numerical models for differential problems, Vol. 2, Springer Science \& Business Media, 2014.

[55] E. Stein, Singular Integrals and Differentiability Properties of Functions, Princeton, University Press, Princeton, N.J., 1970.

[56] A. Quarteroni, R. Sacco, F. Saleri, Numerical mathematics, 2nd Edition, Vol. 37 of Texts in Applied Mathematics, Springer-Verlag, Berlin, 2007.

[57] M. J. Grote, D. Schötzau, Optimal error estimates for the fully discrete interior penalty dg method for the wave equation, Journal of Scientific Computing 40 (1) (2009) 257-272.

[58] G. Cohen, Higher-Order Numerical Methods for Transient Wave Equations, Springer, 2002.

[59] M. Ainsworth, Discrete dispersion relation for hp-version finite element approximation at high wave number, SIAM J. Numer. Anal. 42 (2) (2004) 553-575.

[60] M. Ainsworth, Dispersive and dissipative behaviour of high order discontinuous Galerkin finite element methods, J. Comput. Phys 198 (1) (2004) 106-130.

[61] I. Mazzieri, F. Rapetti, Dispersion analysis of triangle-based spectral element methods for elastic wave propagation, Numer. Algor. 60 (4) (2012) 631-650.

[62] T. Liu, M. K. Sen, T. Hu, J. D. De Basabe, L. Li, Dispersion analysis of the spectral element method using a triangular mesh, Wave Motion 49 (4) (2012) 474-483. 
[63] P. F. Antonietti, C. Marcati, I. Mazzieri, A. Quarteroni, High order discontinuous Galerkin methods on simplicial elements for the elastodynamics equation, Numer. Algor. 71 (1) (2016) $181-206$.

[64] R. Alford, K. Kelly, D. M. Boore, Accuracy of finite-difference modeling of the acoustic wave equation, Geophysics 39 (6) (1974) 834-842.

[65] J. D. De Basabe, M. K. Sen, Grid dispersion and stability criteria of some common finiteelement methods for acoustic and elastic wave equations, Geophysics 72 (6) (2007) T81-T95.

[66] J. D. De Basabe, M. K. Sen, Stability of the high-order finite elements for acoustic or elastic wave propagation with high-order time stepping, Geophys. J. Int. 181 (1) (2010) 577-590.

[67] P. F. Antonietti, P. Houston, A class of domain decomposition preconditioners for hpdiscontinuous Galerkin finite element methods, J. Sci. Comput. 46 (1) (2011) 124-149.

[68] J. M. Carcione, D. Kosloff, R. Kosloff, Wave-propagation simulation in an elastic anisotropic (transversely isotropic) solid, Q. J. Mech. Appl. Math 41 (3) (1988) 319.

[69] J. de la Puente, M. Käser, M. Dumbser, H. Igel, An Arbitrary High Order Discontinuous Galerkin Method for Elastic Waves on Unstructured Meshes IV: Anisotropy, Geophys. J. Int. 169 (3) (2007) 1210-1228.

[70] D. Komatitsch, C. Barnes, J. Tromp, Simulation of anisotropic wave propagation based upon a spectral element method, Geophysics 65 (4) (2000) 1251-1260.

[71] K. Helbig, Handbook of geophysical exploration., Helbig, K. (ed.) Foundations of Anisotropy for Exploration Seismics, volume 22 of Handbook of Geophysical Exploration: Seismic Exploration. Pergamon, Oxford, 1994. 